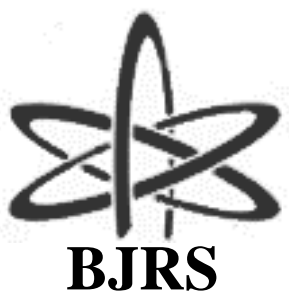

BRAZILIAN JOURNAL

$\mathrm{OF}$

RADIATION SCIENCES

05-02 (2017) 01-29

\title{
Radiological hazard indices and elemental composition of Brazilian and Swiss ornamental rocks
}

\author{
T. M. El Hajja ${ }^{a}$ P. S. C. Silva ${ }^{\text {b }}$ M. P. A. Gandolla ${ }^{c}$, G. A. S. A. Dantas ${ }^{d}$, A. Santos ${ }^{\text {b }}$ \\ and H. Delboni Jr. ${ }^{\mathrm{d}}$ \\ ${ }^{a}$ University of Alfenas, Rodovia José Aurélio Vilela, 11999, Poços de Caldas, Brasil \\ ${ }^{b}$ Institute of Nuclear and Energetic Research, Av. Prof. Lineu Prestes, 2242, São Paulo, Brasil \\ ${ }^{c}$ Università della Svizzera Italiana (USI), Via Giuseppe Buffi, 13, 6900 Lugano, Switzerland \\ ${ }^{d}$ University of São Paulo, Rua Professor Melo Moraes, 2373, São Paulo, Brasil \\ pscsilva@ipen.br
}

\begin{abstract}
The objective of this paper was to evaluate the radiological risk index of ornamental rocks sold both in Brazil and Europe and to correlate their radioactive content with their chemical composition. The ${ }^{238} \mathrm{U},{ }^{232} \mathrm{Th}$ and ${ }^{40} \mathrm{~K}$ mean values were $62 \pm 65,122 \pm 111,1126 \pm 516 \mathrm{~Bq} \mathrm{~kg}^{-1}$ for Brazilian and $93 \pm 59,70 \pm 67$ and $1005 \pm 780 \mathrm{~Bq} \mathrm{~kg}^{-1}$ for Swiss samples, respectively. The radiological index: radium equivalent, external hazard index, absorbed dose rate in air, annual gonadal equivalent dose, annual effective dose equivalent, and excess lifetime cancer risk for Brazilian and Swiss samples were calculated. The main contribution for the radiological indices observed was the radionuclide ${ }^{232} \mathrm{Th}$, which is associated with $\mathrm{REE}, \mathrm{Br}, \mathrm{Hf}, \mathrm{Na}, \mathrm{Rb}, \mathrm{Sb}$ and $\mathrm{Zr}$ in the rock matrix.
\end{abstract}

Keywords: Ornamental rocks, radiological risk index, granite, elemental composition. 


\section{INTRODUCTION}

In Brazil, approximately 80 percent of the exported dimension stones are commercially named granites [1]. This category includes not only the materials meeting the concept of felsic rocks, but also any mafic fine-grained rocks, or other igneous or metamorphic rocks that possess qualities similar to granite's grainy and interlocking texture [2]. In general, rocks generated in the Earth's crust are more enriched in radioelements, e.g. uranium, thorium and radium, relative to those formed in the mantle. This phenomenon is a consequence of magma partial fusion and fractioned crystallization processes that concentrated them in the silica-enhanced liquid phase [3].

The natural radioactivity of the rocks used as building materials has been increasingly studied due to the rising number of homes coated with ornamental rocks. Such aspect has been causing health concerns as an elevation of toxicity risk in these places may occur [3]. The European Union, for example, has a proper legislation regarding natural radioactivity in building materials, which establishes the gamma activity concentration index as an assessment tool [4]. Thus, this paper aims to evaluate the radiological risk index of dimension stones collected both in Brazil and in Switzerland as well as to correlate their radioactive content with their chemical composition based on the concentration of the stable elements.

\section{MATERIALS AND METHODS}

\subsection{Sampling and sample preparation}

The analyzed samples include magmatic and metamorphic rocks of different lithologic types used for decorative purposes that are best sellers in Brazil (20 samples) and Switzerland (14 samples), i.e. diorite, syenite, monzogranite, granitic pegmatites, gneisses, etc. The selected rocks list from Brazil can be found in Fig. 1, and were named according to the Brazilian state acronym where they were collected. Switzerland samples (named CH-sample) are not necessarily mined in this country, but they are easily found for sale in the market and their real provenance was not known for this study. The geographic diversity of the samples is expected to influence the results on the natural radioactivity. The samples preparation included comminution with two different types of crushers, a 
jaw crusher and a roll crusher, and pulverization in a pan to attain a product finer than $0.1 \mathrm{~mm}$. The mass reduction stages were carefully conducted so the final $1 \mathrm{~g}$ sample was not biased.

Figure 1: Brazilian samples provenance identification

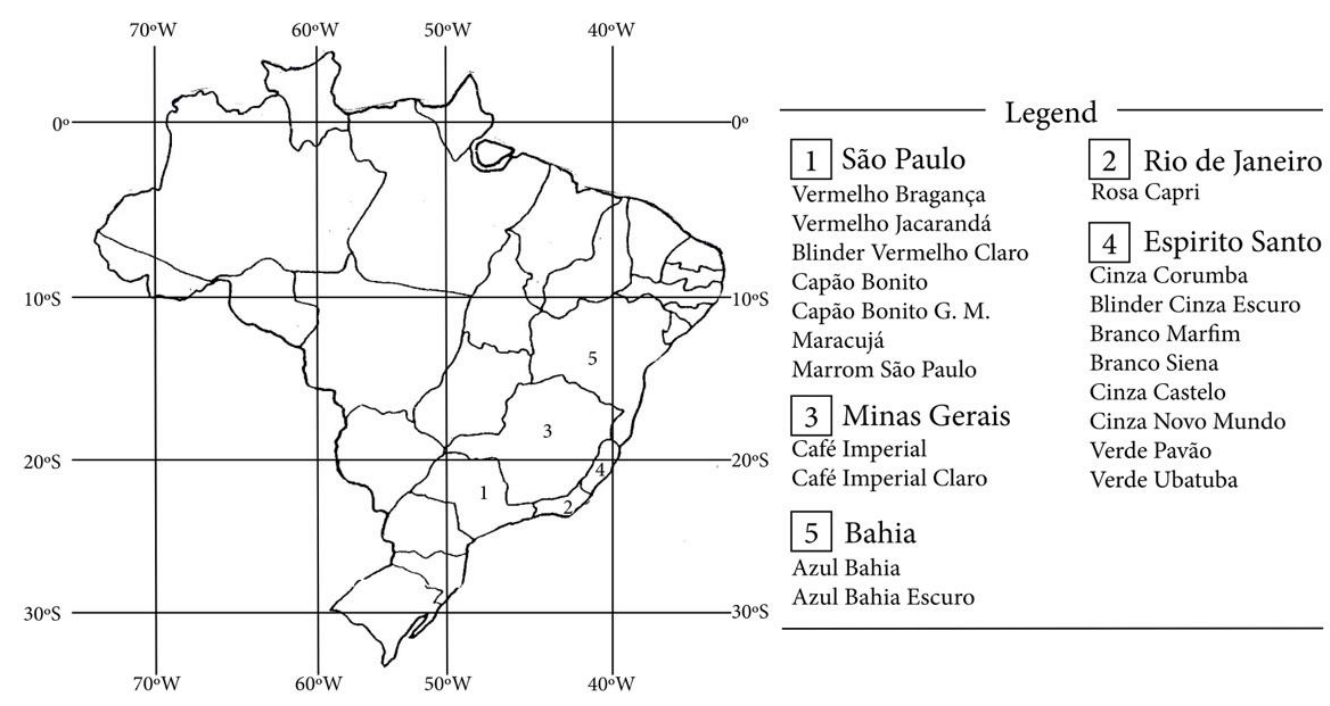

\subsection{Neutron activation analysis measurement}

For the determination of the elemental concentrations, approximately $150 \mathrm{mg}$ of each granite sample were weighed and packed in plastic polyethylene bags. Each batch of samples was irradiated together with two reference materials (RM), USGS STM-2 and NIST SRM 1646a, and a paper filter pipetted with a standard solution of the elements of interest. Each sample was calculated related to each reference material and the final report of the results is the mean value related to each RM. All samples and RM were irradiated for 8 hours in the IEA-R1 research reactor, at IPEN (Instituto de Pesquisas Energéticas e Nucleares) under a thermal neutron flux of $10^{12} \mathrm{~cm}^{-2} \mathrm{~s}^{-1}$. The cooling time for the elements $\mathrm{Na}, \mathrm{K}, \mathrm{As}, \mathrm{Br}, \mathrm{Sb}, \mathrm{La}, \mathrm{Nd}, \mathrm{Sm}, \mathrm{Tb}$ and $\mathrm{U}$ counting varied from 5 to 7 days and for the elements $\mathrm{Ca}, \mathrm{Sc}, \mathrm{Cr}, \mathrm{Fe}, \mathrm{Co}, \mathrm{Zn}, \mathrm{Se}, \mathrm{Rb}, \mathrm{Zr}, \mathrm{Cs}, \mathrm{Ba}, \mathrm{Ce}, \mathrm{Eu}, \mathrm{Yb}, \mathrm{Lu}, \mathrm{Hf}$, Ta and Th was about 15 days.

The counting time was $1 \mathrm{~h}$ for each sample and for the reference material. Gamma spectrometry was performed by using an EG\&G Ortec Ge high pure Gamma Spectrometer detector (AMETEK Inc., USA) and associated electronics, with a resolution of 0.88 and $1.90 \mathrm{keV}$ for ${ }^{57} \mathrm{Co}(122 \mathrm{keV})$ 
and ${ }^{60} \mathrm{Co}(1332 \mathrm{keV})$, respectively [5]. An analysis of the data was carried out by using an in-house gamma ray software, VISPECT program, to identify the gamma-ray peaks. The methodology evaluation was done by cross-checking the reference materials and synthetic standards.

\subsection{Radiological hazard indices}

For the activity concentration of natural uranium, thorium and potassium, their specific activity of 12.437 $\mathrm{Bq} \mathrm{kg}^{-1}, 4.057 \mathrm{~Bq} \mathrm{~kg}^{-1}$ and $262 \mathrm{~Bq} \mathrm{~kg}^{-1}$, respectively, was used considering an isotopic abundance of $99.2742 \%$ for ${ }^{238} \mathrm{U}, 100 \%$ for ${ }^{232} \mathrm{Th}$ and $0.0117 \%$ for ${ }^{40} \mathrm{~K}$ [6].

Radium equivalent activity (Raeq) was defined as the weighted sum of ${ }^{238} \mathrm{U},{ }^{232} \mathrm{Th}$ and ${ }^{40} \mathrm{~K}$ activities, based on the assumption that $370 \mathrm{~Bq} \mathrm{~kg}{ }^{-1}$ of ${ }^{238} \mathrm{U}, 259 \mathrm{~Bq} \mathrm{~kg}^{-1}$ of ${ }^{232} \mathrm{Th}$ and $4810 \mathrm{~Bq} \mathrm{~kg}^{-1}$ of ${ }^{40} \mathrm{~K}$ gives an effective dose of $1.5 \mathrm{mGy}$ per year, equal to $1 \mathrm{mSv}$ annual effective dose [7]. Raeq was calculated from the following relation (Eq. 1) suggested by Beretka and Mathew [8]:

$$
\text { Raeq }=\mathrm{CU}+1.43 \mathrm{CTh}+0.077 \mathrm{CK}
$$

where CU, CTh and $\mathrm{CK}$ are the activity concentrations of ${ }^{238} \mathrm{U},{ }^{232 \mathrm{Th}}$ and ${ }^{40} \mathrm{~K}$, in $\mathrm{Bq} \mathrm{kg}^{-1}$, respectively.

The external hazard index (Hex), proposed by Hewamanna et al. [9], is applied for a house with walls of finite thickness, windows and doors, and can be calculated as Eq. (2):

$$
\mathrm{Hex}=\mathrm{CU} / 740+\mathrm{CTh} / 520+\mathrm{CK} / 9620
$$

The radiation hazard due to Hex will be negligible if its value was less than the unity. Additionally, the internal hazard index (Hin), proposed by Krieger [10], takes into consideration the internal exposure due to radon and its short-lived decay products as a threaten to the respiratory system. It is calculated as Eq. (3):

$$
\text { Hin }=\mathrm{CU} / 185+\mathrm{CTh} / 259+\mathrm{CK} / 4810
$$

The absorbed dose rate (D) is related to the risk due to the amount of radiation deposited in a body per unit of time that arises from terrestrial gamma emitters. $\mathrm{D}$ can be derived $\left(\mathrm{nGy} \mathrm{h}^{-1}\right)$ from the measured activity concentrations and the following conversion factors, as given by UNSCEAR [11] and shown in Eq. (4):

$$
\mathrm{D}=0.462 \mathrm{CU}+0.604 \mathrm{CTh}+0.0417 \mathrm{CK}
$$

The annual gonadal dose equivalent (AGDE) is a measure of the genetic significance of the dose equivalent received by the population's reproductive organs per year [12]. Within this context, the 
activity bone marrow and the bone surface cells were also included by UNSCEAR [13] as organs of interest. The AGDE for the rock samples here analyzed were determined by Eq. $(5)[14,15]$.

$$
\operatorname{AGDE}(\mu \mathrm{Sv} / \mathrm{y})=3.09 \mathrm{CU}+4.18 \mathrm{CTh}+0.314 \mathrm{CK}
$$

The annual effective dose equivalent (AEDE) takes into consideration the adsorbed dose rate and also the time spent in contact with the radioactive source - occupancy factor, according to the formula given by Eq. (6):

$$
\operatorname{AEDE}(\mu \mathrm{Sv} / \mathrm{y})=\mathrm{D}(\mathrm{nGy} / \mathrm{h}) \times 8760 \mathrm{~h} \times 0.8 \times 0.7 \mathrm{~Sv} / \mathrm{Gy} \times 10^{-3}
$$

An occupancy factor of 0.8 , calculated for building materials, is used considering that a person spends $80 \%$ of his time indoors. The conversion factor of $0.7 \mathrm{~Sv} / \mathrm{Gy}$ converts absorbed dose rate (nGy/h) to annual effective dose equivalent in $\mathrm{mSv} / \mathrm{y}[16]$.

Excess life time cancer risk (ELCR) represents the risk of fatal cancer during a life time (DL) of 70 years taking into consideration AEDE and a risk factor $\left(\mathrm{RF}\right.$ in $\left.\mathrm{Sv}^{-1}\right)$ established as 0.05 by the ICRP 60 for stochastic effects for the public [17,18] according to Eq. (7):

$$
\mathrm{ELCR}=\mathrm{AEDE} \times \mathrm{DL} \times \mathrm{RF}
$$

\subsection{Statistical analysis}

Univariate and multivariate statistical analyses were applied to the results for data interpretation. Pearson Correlation Coefficient, in which the correlation coefficient ( $r$ ) is used to measure association strengths, was used to verify the relationship between the natural radionuclide activity concentrations and stable elements [19,20]. Hierarchical cluster analyses were applied with the purpose of assembling objects based on their similarities. This goal is achieved by sorting cases into groups, or clusters, resulting in a strong association between members of the same cluster, and a weak association between members of different clusters [21]. Principal component analysis was used to quantify the significant variation in the data set and to reduce the number of variables to a small number of indices. These indices retain the maximum amount of the variance and result in the retention of only the important characteristics of the original data [22,23]. 


\section{RESULTS AND DISCUSSION}

\subsection{Radiological and elemental characterization}

For quality control of the results, Table 1 shows the certified values of elements present in the reference materials USGS-STM-2, SRM 1642a and the limit of detection. It can be seen that a good agreement was found between the certified values and the measured values for all the determined elements.

Table 1: Certified values (CV) and measured values (MV) obtained in reference materials for quality control of the results and limit of detection (LD). Values in $\mathrm{mg} \mathrm{kg}^{-1}$, except where indicated $(\%)$.

\begin{tabular}{|c|c|c|c|c|c|c|c|c|c|}
\hline & \multicolumn{4}{|c|}{ USGS - STM-2 } & \multicolumn{4}{|c|}{ NIST SRM 1646a } & \multirow{3}{*}{$\begin{array}{l}\text { LD } \\
2.1\end{array}$} \\
\hline & \multicolumn{2}{|c|}{$\mathrm{CV}$} & \multicolumn{2}{|c|}{$\mathrm{MV}$} & \multicolumn{2}{|c|}{$\mathrm{CV}$} & \multicolumn{2}{|c|}{$\mathrm{MV}$} & \\
\hline As & & & & & 6.23 & \pm 0.21 & 6.1 & \pm 0.7 & \\
\hline $\mathrm{K}(\%)$ & 3.38 & \pm 0.17 & 3.1 & \pm 0.8 & 0.864 & \pm 0.016 & 0.9 & \pm 0.4 & 0.11 \\
\hline La & 154 & \pm 11 & 148.1 & \pm 6.7 & 17 & & 18.7 & \pm 0.9 & 0.56 \\
\hline $\mathrm{Na}(\%)$ & 6.61 & \pm 0.38 & 6.4 & \pm 0.2 & 0.741 & \pm 0.017 & 0.74 & \pm 0.03 & 0.021 \\
\hline $\mathrm{Nd}$ & 81 & \pm 4.8 & 84 & \pm 19 & 15 & & 14 & \pm 3 & 3.02 \\
\hline $\mathrm{Sb}$ & & & & & 0.3 & & 0.32 & \pm 0.06 & 0.12 \\
\hline Sm & 12 & \pm 0.9 & 8.6 & \pm 0.8 & & & & & 0.066 \\
\hline U & 7.6 & & 7 & \pm 1 & 2 & & 2.2 & \pm 0.5 & 0.26 \\
\hline $\mathrm{Ba}$ & 639 & \pm 61 & 648 & \pm 74 & 210 & & 230 & \pm 36 & 56 \\
\hline $\mathrm{Ca}(\%)$ & 0.78 & \pm 0.03 & 0.7 & \pm 0.2 & 0.519 & \pm 0.02 & 0.6 & \pm 0.1 & 0.26 \\
\hline $\mathrm{Ce}$ & 253 & \pm 23 & 231 & \pm 14 & 34 & & 35 & \pm 2 & 0.67 \\
\hline Co & & & & & 5 & & 4.9 & \pm 0.2 & 0.17 \\
\hline $\mathrm{Cr}$ & & & & & 40.9 & \pm 1.9 & 43 & \pm 2 & 1.56 \\
\hline Cs & 1.52 & \pm 0.06 & 1.6 & \pm 0.5 & & & & & 0.42 \\
\hline $\mathrm{Eu}$ & 3.45 & \pm 0.25 & 3.4 & \pm 0.3 & & & & & 0.072 \\
\hline $\mathrm{Fe}(\%)$ & 3.77 & \pm 0.09 & 3.7 & \pm 0.1 & 2.008 & \pm 0.039 & 2.03 & \pm 0.06 & 0.011 \\
\hline $\mathrm{Hf}$ & 27 & \pm 0.8 & 28.0 & \pm 0.9 & & & & & 0.18 \\
\hline Lu & 0.6 & \pm 0.04 & 0.60 & \pm 0.05 & & & & & 0.029 \\
\hline $\mathrm{Rb}$ & 114 & \pm 11 & 117 & \pm 14 & 38 & & 38 & \pm 5 & 6.43 \\
\hline Sc & & & & & 5 & & 4.85 & \pm 0.06 & 0.023 \\
\hline $\mathrm{Se}$ & & & & & 0.193 & \pm 0.028 & 0.3 & \pm 0.2 & 0.32 \\
\hline $\mathrm{Ta}$ & 16 & \pm 1.1 & $1 \gamma$ & \pm 1 & & & & & 0.15 \\
\hline $\mathrm{Tb}$ & 1.33 & & 1.3 & \pm 0.2 & & & & & 0.21 \\
\hline Th & 27 & \pm 5 & 29 & \pm 3 & 5.8 & & 5.8 & \pm 0.6 & 0.14 \\
\hline $\mathrm{Yb}$ & 4.2 & \pm 0.8 & 4.3 & \pm 0.7 & & & & & 0.22 \\
\hline $\mathrm{Zn}$ & 223 & \pm 19 & 216 & \pm 16 & & & & & 2.50 \\
\hline $\mathrm{Zr}$ & 1280 & \pm 62 & 1257 & \pm 147 & & & & & 130 \\
\hline
\end{tabular}


Table 2 shows the commercial name, provenance, sample code, petrographic classification and activity concentrations (AC) for ${ }^{232} \mathrm{Th},{ }^{238} \mathrm{U}$ and ${ }^{40} \mathrm{~K}$, in $\mathrm{Bq} \mathrm{kg}^{-1}$. The uncertainty of the results was obtained by means of error propagation for $95 \%$ of confidence. Considering all the samples, thorium, uranium and potassium AC showed a range from 2.5 to $2966 \mathrm{~Bq} \mathrm{~kg}^{-1}, 3.9$ to $214 \mathrm{~Bq} \mathrm{~kg}^{-1}$ and 25 to $2372 \mathrm{~Bq} \mathrm{~kg}^{-1}$, respectively. The highest values of activity concentrations were found for Th in sample CH7, from Switzerland; for U, in the sample RJ1 from Rio de Janeiro, Brazil; and for ${ }^{40} \mathrm{~K}$, in the sample MG2, from Minas Gerais, Brazil.

A comparison of the AC according to the samples' place of origin is shown in Fig. 2. Among the Brazilian samples, the ones from Minas Gerais (MG) presented the highest ${ }^{40} \mathrm{~K}$ value. Despite the fact that only one sample from Rio de Janeiro (RJ) was analyzed, it showed the highest ${ }^{238} \mathrm{U}$ content and presented almost the same value as the mean concentration of São Paulo (SP) samples for ${ }^{232} \mathrm{Th}$. The mean AC and standard deviation for all Brazilian and Swiss samples were: $62 \pm 55 \mathrm{~Bq}$ $\mathrm{kg}^{-1}$ and $93 \pm 60 \mathrm{~Bq} \mathrm{~kg}^{-1}$ for ${ }^{238} \mathrm{U} ; 1126 \pm 516 \mathrm{~Bq} \mathrm{~kg}^{-1}$ and $1005 \pm 778 \mathrm{~Bq} \mathrm{~kg}^{-1}$ for ${ }^{40} \mathrm{~K} ; 122 \pm 111$ $\mathrm{Bq} \mathrm{kg}^{-1}$ and $293 \pm 806 \mathrm{~Bq} \mathrm{~kg}^{-1}$ for ${ }^{232} \mathrm{Th}$, respectively. These values indicate that a wide range of radionuclides $\mathrm{AC}$ can be found in the analyzed ornamental rocks. The high mean and standard deviation for ${ }^{232} \mathrm{Th}$ in the Swiss samples were due to the value found in sample $\mathrm{CH} 7$. If not taking this sample in consideration, the mean value and standard deviation become $70 \pm 67 \mathrm{~Bq} \mathrm{~kg}^{-1}$ for this radionuclide, lower than the Brazilian mean AC.

The differences observed in the activity concentrations are not just related to the provenance of the rocks, but mainly to their mineralogical composition. Elements with a lithophile behavior such as $\mathrm{U}$, Th and $\mathrm{K}$ concentrate in the Earth`s crust during the melting in the mantle and basalt differentiation being accumulated in felsic igneous melts [24]. Higher concentrations of $U$ are generally found in acid igneous or granitic rocks while basaltic rocks contain lower concentration [25]. Thorium is 3 to 4 times more abundant than uranium in the rocks of the lithosphere [26] and sedimentary rocks generally contain only few $\mu \mathrm{g} \mathrm{g}^{-1}$ of this element. On the other hand, its concentration can be up to 10 times higher in acid igneous rocks, where it can substitute the rare earth elements [27].

Fig. 3 shows the $\mathrm{Th} / \mathrm{U}$ ratio in the samples. It can be seen that the ornamental rocks from Brazil tend to be enriched in Th compared to the Swiss samples. This ratio is closer to the global average (3 to 4) in the $\mathrm{CH}$ samples while most of the Brazilian ones present this ratio in the range of 5 to 15 . 
The high values of Th/U ratio in the São Paulo samples are probably due to an enrichment in Th as their concentrations are generally higher than mean values found in granites [28].

Table 2: Activity concentration of ${ }^{232} \mathrm{Th},{ }^{238} \mathrm{U}$ and ${ }^{40} \mathrm{~K}$, in $\mathrm{Bq} \mathrm{kg}^{-1}$, of the analyzed samples, their commercial names and petrographic classification.

\begin{tabular}{|c|c|c|c|c|c|c|c|}
\hline $\begin{array}{l}\text { Sample } \\
\text { ID }\end{array}$ & Commercial name & $\begin{array}{l}\text { Petrographic } \\
\text { classification }\end{array}$ & $\begin{array}{l}232 \\
\mathrm{~Bq}\end{array}$ & & $\begin{array}{c}2{ }^{238} \mathrm{U} \\
\mathrm{Bq} / \mathrm{kg}\end{array}$ & $\begin{array}{l}{ }^{40} \mathrm{~F} \\
\mathrm{~Bq} /\end{array}$ & \\
\hline CH1 & Rosso Lucido & SGR & & \pm 4 & $64 \pm 4$ & 1674 & \pm 493 \\
\hline $\mathrm{CH} 2$ & Iragna & GRN & 97 & \pm 5 & $101 \pm 7$ & 1551 & \pm 463 \\
\hline $\mathrm{CH} 3$ & Basaltito & BST & 240 & \pm 14 & $169 \pm 9$ & 1797 & \pm 86 \\
\hline $\mathrm{CH} 4$ & Branco Suiça & BGM & 34.9 & \pm 2.1 & $19 \pm 2$ & 558 & \pm 31 \\
\hline CH5 & Flor di Pesco & MRB & 37.0 & \pm 2.2 & $144 \pm 7$ & 31 & \pm 7 \\
\hline CH6 & Bianco Cristal & MGR & 85 & \pm 5 & $181 \pm 17$ & 1306 & \pm 229 \\
\hline $\mathrm{CH} 7$ & Sodalita & NSY & 2966 & \pm 174 & $<\mathrm{LD}$ & $<\mathrm{LD}$ & \\
\hline CH8 & Piettra Lavica & ADT & 19.3 & \pm 1.1 & $86 \pm 6$ & 447 & \pm 43 \\
\hline CH9 & Pietra Lavica (1) & ADT & 3.87 & \pm 0.25 & $<\mathrm{LD}$ & $<\mathrm{LD}$ & \\
\hline $\mathrm{CH} 10$ & Pietra de Jerusalem & MRB & 2.47 & \pm 0.15 & $32 \pm 2$ & 25 & \pm 18 \\
\hline CH11 & Onsernone Lucido & GRN & 53.9 & \pm 3.0 & $65 \pm 5$ & 201 & \pm 116 \\
\hline $\mathrm{CH} 12$ & Mônaco Light & MRB & 49.5 & \pm 3.0 & $16 \pm 1$ & 2213 & \pm 671 \\
\hline $\mathrm{CH} 13$ & Lusera Fiammata & MGC & 143 & \pm 9 & $146 \pm 3$ & 1250 & \pm 368 \\
\hline SP1 & Capão Bonito Grãos & MGR & 356 & \pm 20 & $46 \pm 3$ & 599 & \pm 177 \\
\hline SP2 & Capão Bonito & MGR & 387 & \pm 21 & $131 \pm 8$ & 1182 & \pm 348 \\
\hline SP3 & Vermelho Bragança & MGR & 169 & \pm 10 & $94 \pm 5$ & 1186 & \pm 65 \\
\hline SP4 & Maracujá & MGR & 282 & \pm 16 & $24 \pm 6$ & 1125 & \pm 73 \\
\hline SP5 & Vermelho Jacarandá & SGR & 147 & \pm 8 & $47 \pm 5$ & 223 & \pm 106 \\
\hline SP6 & Marrom São Paulo & SGR & 122 & \pm 7 & $73 \pm 5$ & 1385 & \pm 80 \\
\hline SP7 & Blinder Vermelho Claro & MGR & 131 & \pm 8 & $52 \pm 3$ & 1246 & \pm 65 \\
\hline ES1 & Blinder Cinza Escuro & QMZ & 14.4 & \pm 0.9 & $4 \pm 2$ & 1041 & \pm 191 \\
\hline ES2 & Branco Siena & SGR & 44.1 & \pm 2.5 & $10 \pm 2$ & 871 & \pm 60 \\
\hline ES3 & Cinza Corumbá & MGR & 66.0 & \pm 3.8 & $83 \pm 4$ & 1326 & \pm 84 \\
\hline ES4 & Cinza Castelo & MGR & 82 & \pm 4 & $18 \pm 3$ & 962 & \pm 51 \\
\hline ES5 & Branco Marfim & SGR & 73 & \pm 4 & $25 \pm 3$ & 1365 & \pm 76 \\
\hline ES6 & Verde Pavão & QMZ & 11.2 & \pm 0.6 & & 1275 & \pm 75 \\
\hline ES7 & Cinza Novo Mundo & SGR & 104 & \pm 5 & $19 \pm 2$ & 1333 & \pm 66 \\
\hline ES8 & Verde Ubatuba & QMZ & 17.9 & \pm 1.0 & $22 \pm 3$ & 549 & \pm 33 \\
\hline BA1 & Azul Bahia & FMZ & 22.6 & \pm 1.3 & $146 \pm 9$ & 1694 & \pm 515 \\
\hline BA2 & Azul Bahia Escuro & FMZ & 80 & \pm 5 & $100 \pm 6$ & 772 & \pm 53 \\
\hline MG1 & Café Imperial & AFS & 49.3 & \pm 3.0 & $65 \pm 13$ & 1797 & \pm 342 \\
\hline MG2 & Café Imperial Claro & AFS & 51.5 & \pm 3.2 & $16 \pm 1$ & 2372 & \pm 703 \\
\hline RJ1 & Rosa Capri & SGR & 230 & \pm 13 & $214 \pm 16$ & 1384 & \pm 81 \\
\hline
\end{tabular}

SGR: Syenogranite; MGR: monzogranite; FMZ: foid-monzodiorite; GRN: granodiorite; BST: basaltite; BGM: biotite gneiss with muscovite; MRB: marble; QMZ: quartz monzodiorite; NSY: 
nepheline syenite; AFS: alkali feldspar syenite; ADT: andesite; MGC: mylonitic gneiss with chlorite and muscovite.

Figure 2: Mean activity concentration according to the sample origin: CH: Switzerland, BR: Brazil. SP, ES, BA, MG and RJ stands for the Brazilian states of São Paulo, Espírito Santo, Bahia, Minas Gerais and Rio de Janeiro, respectively

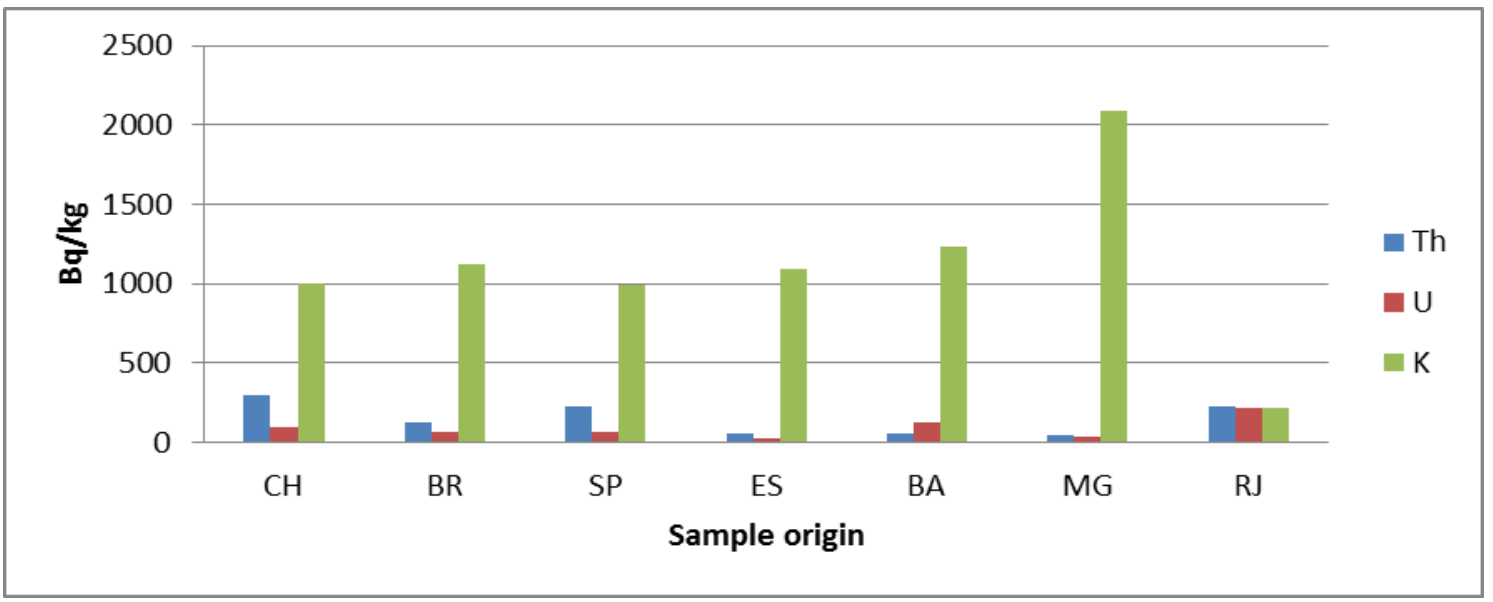

Figure 3: Th/U ratio in the analyzed samples

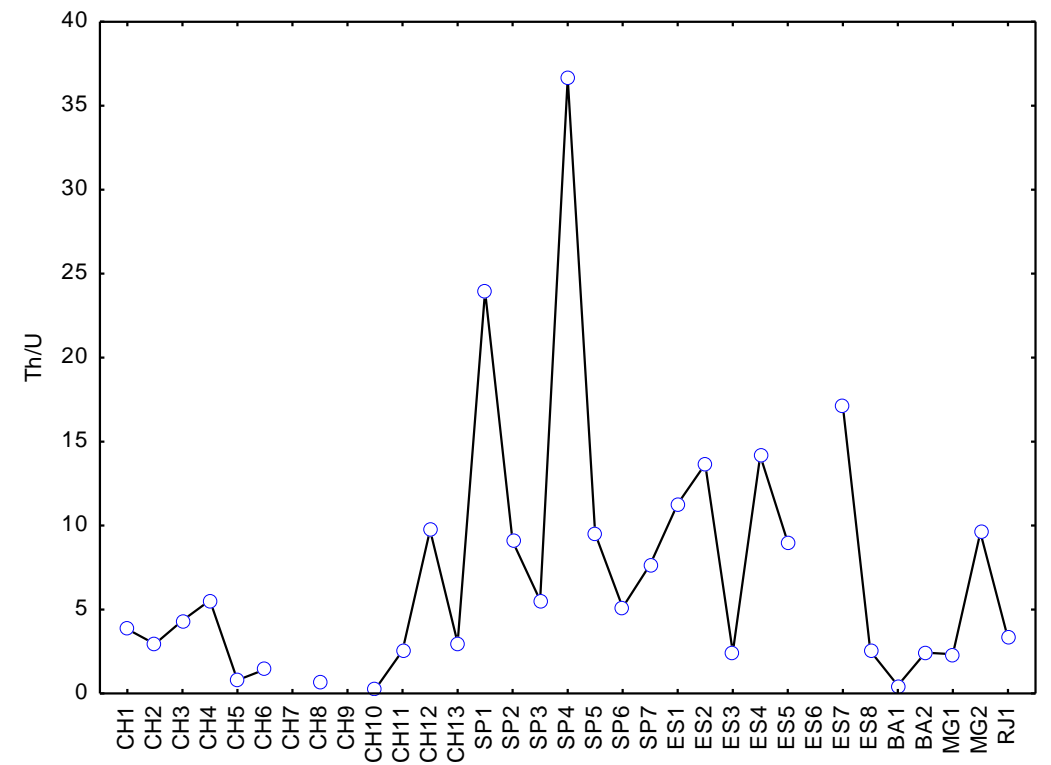


For chemical characterization of the ornamental rock samples, trace elements were also determined and their concentrations are shown in Table 3. Table 4 shows the Pearson correlation coefficients obtained for these results. According to the results, Th showed a good correlation with rare earth elements (REE), Br, Hf, $\mathrm{Na}, \mathrm{Rb}, \mathrm{Sb}$ and $\mathrm{Zr}$; uranium was well correlated only with $\mathrm{Cs}$; and $\mathrm{K}$ presented good correlations with $\mathrm{Ba}, \mathrm{REE}, \mathrm{Zr}$ and a negative correlation with $\mathrm{Ca}$. The good correlation among $\mathrm{Th}, \mathrm{Zr}$, and REE may indicate that these elements are probably associated with minerals such as zircon and apatite $[29,30]$. The elevated value of Th, REE and $\mathrm{Zr}$ found in sample CH7 matches with the nepheline syenite mineralogy, probably due to the presence of apatite-like minerals, monazite and zirconosilicates [31].

Cluster analysis (CA) applied to the variables, Fig. 4, also shows the Th association with REE, Hf and $\mathrm{Zr}$, also indicating a common origin for these elements, e. g., present in the same minerals constituents of the rocks. Potassium and $U$, on the other hand, are associated with elements such as $\mathrm{Ca}, \mathrm{Co}, \mathrm{Zn}$ and Fe. Fig. 5 shows the loading factors, obtained by factor analysis for the determined elements. The result agreed with the one obtained by CA and shows a high loading factor for Th and REE in the first factor, while $\mathrm{U}$ and $\mathrm{K}$, present low loading factors for both, factor 1 and 2 , being negative for $\mathrm{U}$. This results indicates that the mineral bearing Th must be distinct from that bearing $\mathrm{U}$ and $\mathrm{K}$. In addition to the uranium-bearing minerals, this element can be present in rocks by isomorphic substitution of calcium [32], what could cause the weak inverse loading factor for these elements in factor 2.

The influence of elemental composition of the analyzed rocks is highlighted in Fig. 6 in which clusters formed according to the rock type can be seen. Generally, rocks of the same petrographic classification are grouped together independently of their provenance.

\subsection{Radiological risk assessment}

The radiological hazard index radium equivalent activity (Raeq), external hazard index (Hex), internal hazard index (Hin), absorbed dose rate (D), annual gonadal dose equivalent (AGDE), annual effective dose equivalent (AEDE) and excess life time cancer risk (ELCR) obtained for the samples analyzed in this study are shown in Table 5. 
Table 3: Concentrations and uncertainties of trace elements, in $\mathrm{mg} \mathrm{kg}^{-1}$, except where indicated \%, determined in the analyzed samples.

\begin{tabular}{|c|c|c|c|c|c|c|c|c|c|c|c|c|c|c|c|c|c|c|c|c|c|c|c|c|c|c|}
\hline & As & \pm & $\mathrm{Ba}$ & \pm & $\mathrm{Br}$ & \pm & $\mathrm{Ca}$ & \pm & $\mathrm{Ce}$ & \pm & $\mathrm{Co}$ & \pm & $\mathrm{Cr}$ & \pm & $\mathrm{Cs}$ & \pm & $\mathrm{Eu}$ & \pm & $\mathrm{Fe}$ & \pm & $\mathrm{Hf}$ & \pm & $\mathrm{La}$ & \pm & $\mathrm{Lu}$ & \pm \\
\hline & & & & & & & $\%$ & & & & & & & & & & & & $\%$ & & & & & & & \\
\hline CH1 & 7.6 & 0.4 & 776 & 38 & 8.5 & 0.3 & 0.23 & 0.03 & 57 & 2 & 0.38 & 0.03 & 69 & 2 & 5.1 & 0.2 & 0.77 & 0.02 & 0.778 & 0.008 & 5.90 & .08 & 27.1 & 0.5 & 0.78 & 0.03 \\
\hline $\mathrm{CH} 2$ & & & 2638 & 138 & 5.5 & 0.3 & 1.13 & 0.09 & 90 & 3 & 4.65 & 0.07 & 495 & 13 & 4.1 & 0.2 & 0.72 & 0.02 & 2.75 & 0.03 & 6.24 & 0.09 & 25.3 & 0.4 & 8.0 & 0.3 \\
\hline $\mathrm{CH} 3$ & 7.6 & 0.4 & 1124 & 57 & $<\mathrm{LD}$ & & 2.5 & 0.2 & 177 & 6 & 8.1 & 0.1 & 83 & 2 & 37 & 3 & 2.46 & 0.07 & 3.61 & 0.03 & 9.3 & 0.1 & 109 & 2 & 0.39 & 0.02 \\
\hline $\mathrm{CH} 4$ & $<\mathrm{LD}$ & & 417 & 22 & $<\mathrm{LD}$ & & 1.3 & 0.1 & 47 & 2 & 3.76 & 0.06 & 159 & 4 & 1.7 & 0.1 & 0.94 & 0.03 & 1.75 & 0.02 & 4.04 & 0.06 & 26.8 & 0.5 & 0.16 & 0.01 \\
\hline CH5 & 0.5 & 0.2 & 70 & 7 & 4.1 & 0.3 & 27 & 2 & 36 & 1 & 0.44 & 0.03 & 20.9 & 0.6 & $<\mathrm{LD}$ & & 0.15 & 0.01 & 0.165 & 0.003 & 0.23 & 0.02 & 22.9 & 0.4 & 0.061 & 0.004 \\
\hline CH6 & 1.8 & 0.3 & 188 & 20 & 3.5 & 0.3 & 0.7 & 0.1 & 29 & 1 & 1.60 & 0.09 & 185 & 6 & 17 & 2 & 0.33 & .02 & 0.79 & 0.01 & 3.83 & .08 & 12.3 & .2 & 0.65 & 0.03 \\
\hline $\mathrm{CH} 7$ & $<\mathrm{LD}$ & & 1401 & 101 & 227 & 2 & 4.4 & 0.6 & 609 & 21 & 4.3 & 0.2 & 113 & 4 & $<\mathrm{LD}$ & & 29.0 & 0.9 & 4.08 & 0.06 & $<\mathrm{LD}$ & & 302 & 5 & $<\mathrm{LD}$ & \\
\hline $\mathrm{CH} 8$ & 53 & 2 & 820 & 57 & $<\mathrm{LD}$ & & 9.1 & 0.3 & 64 & 2 & 46.0 & 0.9 & 231 & 7 & $<\mathrm{LD}$ & & 2.18 & 0.07 & 7.97 & 0.08 & 5.23 & 0.08 & 35.1 & 0.7 & 0.25 & 0.02 \\
\hline $\mathrm{CH} 9$ & $<\mathrm{LD}$ & & 181 & 27 & $<\mathrm{LD}$ & & 3.6 & 0.2 & 24 & 1 & 30.2 & 0.3 & 254 & 7 & $<\mathrm{LD}$ & & 1.74 & 0.04 & 5.64 & 0.05 & 0.92 & 0.06 & 17.4 & 0.1 & 0.17 & 0.02 \\
\hline CH10 & $<\mathrm{LD}$ & & 24 & 9 & 1.7 & 0.2 & 32 & 2 & 5.6 & 0.3 & 5.78 & 0.06 & 319 & 9 & 0.09 & 0.06 & 0.076 & 0.005 & 0.540 & 0.006 & 0.16 & 0.03 & 3.74 & 0.08 & 0.027 & 0.003 \\
\hline CH11 & $<\mathrm{LD}$ & & 1221 & 5 & 0.0 & 0.4 & 2.9 & & 77 & 4 & 5.2 & & 639 & 17 & 1.6 & .2 & 1.28 & 0.05 & 3.57 & 3 & 6.69 & 08 & 63.8 & .4 & 0.27 & 0.01 \\
\hline CH12 & 1.6 & 0.3 & 4773 & 189 & $<\mathrm{LD}$ & & 0.17 & 0.03 & 338 & 11 & 17.3 & 0 & 155 & 4 & 2.7 & 0.2 & 6.4 & & 92 & 3 & 7.19 & 0.09 & 162 & 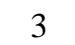 & .26 & .01 \\
\hline CH13 & 3.1 & 0.2 & 174 & 14 & $<\mathrm{LD}$ & & 0.61 & 0.02 & 85 & 3 & 1.46 & 0.06 & 289 & 8 & 9.3 & 0.3 & 0.205 & 0.008 & 1.25 & 01 & 6.78 & 0.08 & 37.6 & 0.7 & 1.20 & 0.05 \\
\hline SP1 & 0.4 & 0.1 & 2769 & 140 & 4.9 & 0.2 & 0.78 & 0.06 & 318 & 10 & 3.12 & 0.06 & 692 & 17 & 3.9 & 0.2 & 0.69 & 0.02 & 2.76 & 0.02 & 12.9 & 0.2 & 73 & 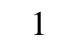 & 16.6 & 0.6 \\
\hline SP2 & 0.27 & 0.06 & 2320 & 124 & 7.7 & 0.1 & 0.74 & 0.06 & 295 & 10 & 2.73 & 0.05 & 594 & 15 & 6.9 & 0.3 & 0.98 & 0.03 & 2.87 & 0.03 & 12.9 & 0.2 & 76 & 1 & 15.3 & 0.5 \\
\hline SP3 & $<\mathrm{LD}$ & & 517 & 27 & 1.9 & 0.5 & 0.85 & 0.08 & 156 & 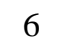 & 2.53 & 0.06 & 154 & 4 & 4.1 & 0 & 0 & 0.03 & 1.51 & 0 & 7.31 & 0.09 & 90 & & 0.39 & 0.02 \\
\hline SP4 & 1.7 & 0.1 & 1289 & 88 & $<\mathrm{LD}$ & & 1.54 & 0.08 & 286 & 9 & 9.2 & & 222 & 1 & 0.5 & 0.2 & I & 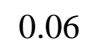 & 2.18 & & 10.2 & 0.1 & 168 & & 0.33 & 0.02 \\
\hline SP5 & $<\mathrm{LD}$ & & 584 & 47 & 39.4 & 0.4 & 0.84 & 0.05 & 110 & 5 & 4.39 & 0.06 & 496 & 13 & 3.4 & 0.2 & 1.46 & 4 & 1.69 & 0.02 & 5.87 & 0.07 & 52.4 & 0.4 & 0.222 & 0.009 \\
\hline SP6 & 1.0 & 0.3 & 875 & 68 & $<\mathrm{LD}$ & & 0.68 & 0.05 & 154 & 8 & 3.18 & 0.05 & 353 & 9 & 2.5 & 0.2 & 1.36 & & 2.06 & 0.02 & 10.1 & 0.1 & 83.8 & 0.5 & 0.99 & 0.03 \\
\hline SP7 & $<\mathrm{LD}$ & & 726 & 38 & 3.0 & 0.5 & 0.76 & 0.07 & 119 & 4 & 3.27 & 0.08 & 294 & 8 & 2.1 & 0.2 & 0.93 & 0.03 & 1.70 & 0.02 & 7.3 & 0.1 & 74 & 9 & 0.58 & 0.02 \\
\hline ES1 & $<\mathrm{LD}$ & & 3358 & 225 & $<\mathrm{LD}$ & & 2.9 & 0.4 & 123 & 4 & 17.7 & 0.3 & 51 & 2 & 2.9 & 0.5 & 2.83 & 0.09 & 6.41 & & 4.7 & 0.1 & 62 & 1 & 0.25 & 0.02 \\
\hline $\mathrm{ES} 2$ & $<\mathrm{LD}$ & & 623 & 47 & $<\mathrm{LD}$ & & 1.72 & 0.08 & 65 & 0 & & 0 & 365 & 11 & $<\mathrm{LD}$ & & 0 & & 1. & & 4.21 & 0.06 & 33.8 & 0.7 & 0.095 & 0.009 \\
\hline ES3 & 5.5 & 0.4 & 1193 & 84 & $<\mathrm{LD}$ & & 2.29 & 0.10 & 116 & 4 & 7.1 & 0.2 & 245 & 8 & 7.3 & 0.3 & 1.55 & 0.05 & 3.13 & 0 & 8.27 & 0.09 & 59 & & 0.42 & 0.02 \\
\hline ES4 & $<\mathrm{LD}$ & & 2914 & 257 & 136 & 2 & 3.0 & 0.1 & 291 & 9 & 365 & 3 & 13.6 & 0.6 & 1.4 & 0.1 & 3.5 & & 4.14 & 0.03 & 15.0 & 0.2 & 151 & J & 0.31 & 0.02 \\
\hline ES5 & 21 & 1 & 413 & 41 & $<\mathrm{LD}$ & & 1.10 & 0.07 & 56 & 2 & 474 & 4 & 10.4 & 0.5 & 1.9 & 0.1 & 0.82 & 0.03 & 1.67 & 0.01 & 4.88 & 0.06 & 24.3 & 0.4 & 0.32 & 0.02 \\
\hline ES6 & $<\mathrm{LD}$ & & 3180 & 284 & $<\mathrm{LD}$ & & 3.1 & 0.2 & 156 & 5 & 93.6 & 0.8 & 13.5 & 0.5 & $<\mathrm{LD}$ & & 4.0 & & 4.71 & 0.04 & 16.5 & 0.2 & 77 & 1 & 0.34 & 0.02 \\
\hline ES7 & $<\mathrm{LD}$ & & 815 & 81 & $<\mathrm{LD}$ & & 1.34 & 0.07 & 117 & 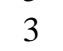 & 151 & 1 & 11.0 & 0.4 & 1.4 & & 0.97 & & 2.32 & & 11.8 & .1 & 53.8 & 0.9 & 0.40 & 0.02 \\
\hline ES8 & $<\mathrm{LD}$ & & 1828 & 162 & 1.6 & 0.7 & 4.6 & 0 & 142 & 4 & 23.4 & & 423 & 11 & 0.23 & 0.06 & & & 5.32 & & 5.97 & 0.07 & 78 & 1 & 0.178 & 0.008 \\
\hline BA1 & 3.4 & 0.3 & 138 & 9 & 91.9 & 0.8 & 0.56 & 0.04 & 28.4 & 0.9 & 0.75 & 0.03 & 99 & 3 & 5.0 & 0.2 & 0.12 & 0.01 & 1.44 & 0.01 & 11.9 & 0.1 & 14.2 & 0.3 & 0.26 & 0.01 \\
\hline BA2 & 8.7 & 0.5 & 222 & 22 & 103 & 2 & 0.51 & 0.05 & 47 & 2 & 3.2 & 0.1 & 55 & 2 & 7.0 & 0.3 & 0.34 & 0.01 & 2.09 & 0.02 & 20.2 & 0.2 & 25.5 & 0.5 & 0.27 & 0.01 \\
\hline MG1 & 3.8 & 0.8 & 5874 & 392 & $<\mathrm{LD}$ & & 2.3 & 0.3 & 287 & 10 & 15.2 & 03 & 133 & 4 & 3.4 & 0.6 & 8.2 & 0.2 & 4.11 & 0.06 & 7.4 & 0.2 & 144 & 3 & 0.42 & 0.03 \\
\hline MG2 & 1.7 & 0.3 & 4981 & 197 & $<\mathrm{LD}$ & & 0.18 & 0.03 & 352 & 12 & 18.0 & 0 & 161 & 4 & 2.8 & 0.2 & 6.6 & 0.2 & 4.09 & 0.04 & 7.49 & 0.09 & 173 & 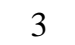 & 0.27 & 0.01 \\
\hline RJ1 & 3.3 & 0.7 & 1074 & 72 & $<\mathrm{LD}$ & & 1.37 & 0.08 & 198 & 7 & 5.4 & 0.2 & 150 & 5 & 1.30 & 0.07 & 1.40 & 0.04 & 2.10 & 0.02 & 8.14 & 0.09 & 113 & 2 & 0.33 & 0.01 \\
\hline
\end{tabular}




\begin{tabular}{|c|c|c|c|c|c|c|c|c|c|c|c|c|c|c|c|c|c|c|c|c|c|c|c|c|}
\hline & $\mathrm{Na}$ & \pm & $\mathrm{Nd}$ & \pm & $\mathrm{Rb}$ & \pm & $\mathrm{Sb}$ & \pm & $\mathrm{Sc}$ & \pm & $\mathrm{Se}$ & \pm & $\mathrm{Sm}$ & \pm & $\mathrm{Ta}$ & \pm & $\mathrm{Tb}$ & \pm & $\mathrm{Yb}$ & \pm & $\mathrm{Zn}$ & \pm & $\mathrm{Zr}$ & \pm \\
\hline & $\%$ & & & & & & & & & & & & & & & & & & & & & & & \\
\hline CHI & 1.67 & .04 & 19 & 1 & 235 & 11 & 1.01 & 0.00 & 11.00 & 0.00 & 0.7 & 0.2 & 0.15 & .01 & 1.64 & 0.06 & 1.51 & 0.00 & 4.1 & 0.4 & 0595 & 302 & 217 & 19 \\
\hline $\mathrm{CH} 2$ & 2.5 & & 16 & 1 & 612 & 28 & 0.13 & 0.02 & 7.28 & & 1.1 & 0.3 & 0.24 & & 1.66 & 0.06 & 0.98 & 0.07 & 4.2 & 0.3 & 84 & 5 & 361 & 25 \\
\hline $\mathrm{CH} 3$ & 1.99 & 0.05 & 81 & 5 & 434 & 22 & 1.2 & 01 & 4.87 & 0.03 & $<\mathrm{LD}$ & & 21 & 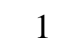 & 1.32 & 0.08 & 1.10 & 0.08 & 2.5 & 0.2 & 81 & & 573 & 28 \\
\hline $\mathrm{CH} 4$ & 3.29 & 0.08 & 21 & 2 & 68 & 4 & 0.20 & 0.04 & 4.66 & 0.03 & $<\mathrm{LD}$ & & 1.41 & 0.07 & 0.65 & 0.04 & 0.34 & 0.04 & 0.87 & 0.08 & 71 & 4 & 193 & 15 \\
\hline CH5 & 0.051 & 0.002 & 10 & 2 & 5.1 & 0.9 & $<\mathrm{LD}$ & & 0.55 & 0.01 & 31.2 & 0.4 & 3.7 & 0.2 & 41 & 1 & 0.33 & 0.04 & $<\mathrm{LD}$ & & 26 & 2 & $<\mathrm{LD}$ & \\
\hline CH6 & 2.24 & 0.05 & 19 & 3 & 228 & 14 & $<\mathrm{LD}$ & & 3.45 & 0.03 & 2.1 & 0.6 & 6.6 & 0.3 & 2.2 & 0.1 & 1.4 & 0.1 & 3.2 & 0.2 & 44 & 4 & 166 & 26 \\
\hline $\mathrm{CH} 7$ & 12.2 & 0.3 & 318 & 37 & $<\mathrm{LD}$ & & 3 & 1 & 11.47 & 0.08 & $<\mathrm{LD}$ & & 98 & 4 & 2.7 & 0.2 & 12 & 1 & 5.3 & 0.5 & 906 & 52 & 1733 & 103 \\
\hline $\mathrm{CH} 8$ & 2.93 & 0.07 & 27 & 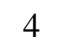 & 27 & 2 & 0.18 & 0.03 & 19.8 & 01 & 23 & 1 & 6.3 & 0.2 & 24.7 & 0.8 & 1.9 & 0.4 & 1.8 & 0.3 & 105 & & 156 & 73 \\
\hline CH9 & 2.39 & 6 & 12 & 2 & 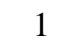 & 1 & $<\mathrm{LD}$ & & 14.49 & & 0.3 & 0.7 & 4.4 & & 0 & 0.1 & 0.4 & & .3 & 0.2 & & & 61 & 48 \\
\hline $\mathrm{CH} 10$ & 0.023 & 01 & 3 & 2 & $<\mathrm{LD}$ & & 0.15 & 0.02 & 0.432 & 07 & 21.5 & 0.6 & 0.57 & 2 & 18.1 & 0.4 & 0.25 & 4 & .8 & . & 4 & & 7 & 9 \\
\hline CH11 & 2.84 & 0.07 & 28 & 9 & 80 & 3 & 0.73 & 0.09 & 10.71 & 0.05 & 45 & 1 & 9.1 & 0.3 & 51 & 1 & 0.3 & 0 & 3.0 & 0.4 & 148 & 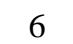 & 295 & 40 \\
\hline CH12 & 2.36 & 0.05 & 150 & 6 & 178 & 10 & 0.24 & 0.02 & 8.99 & 0.04 & 0.0 & 0.3 & 26 & 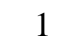 & 1.93 & 0.09 & 1.62 & 0.06 & 1.8 & 0.2 & 102 & 4 & 533 & 17 \\
\hline CH13 & 2.66 & 0.06 & 42 & 2 & 336 & 18 & 0.35 & 0.02 & 2.35 & 0.01 & 2.7 & 0.2 & 11.7 & 0.4 & 3.9 & 0.1 & 2.23 & 0.06 & 8.1 & 0.9 & 64 & 2 & 214 & 10 \\
\hline SP1 & 1.15 & 02 & 94 & 6 & 724 & 32 & $<\mathrm{LD}$ & & 4.03 & 0 & 2.7 & 0.6 & & & 4.9 & 0 . & 2.0 & 0 & 8.6 & 0 & 8 & & 373 & 21 \\
\hline SF & 4 & 0 & 113 & 6 & 969 & 43 & 0.20 & 0.02 & 4.40 & & 3.6 & 0.8 & 11.43 & $H$ & 8.0 & & 2.1 & & & & 89 & & 475 & 25 \\
\hline SP3 & 2.24 & 5 & 75 & 9 & 298 & 15 & $<\mathrm{LD}$ & & 4.00 & 0.02 & $<\mathrm{LD}$ & & 17.8 & 0.9 & 1.01 & 0.06 & 1.05 & 0.07 & 2.1 & 0 & 52 & 3 & 271 & 17 \\
\hline SP4 & 2.21 & 0.05 & 118 & 15 & 220 & 10 & 0.06 & 0.01 & 4.66 & 0.03 & 7.8 & 0.3 & 14.2 & 0 & 8.8 & 0.3 & 1.4 & 0. & 2.0 & 0.3 & 53 & 3 & 403 & 36 \\
\hline SP5 & 1.71 & 0.04 & 49 & 7 & 350 & 12 & 0.38 & 0.07 & 3.90 & & 9.6 & 0.8 & 5.7 & 0.2 & 8.6 & 0.2 & 0.62 & 0.07 & 1.3 & 0.2 & 48 & 2 & 230 & 19 \\
\hline SP6 & 2.92 & 0.07 & 70 & 4 & 298 & 11 & 0.20 & 0.02 & 5.21 & 0 . & 3.5 & 0.4 & 14.3 & 0 . & 4.0 & 0 & 1.68 & 0.08 & 5.8 & 0. & 84 & 4 & 387 & 16 \\
\hline $\mathrm{S}$ & 99 & & 53 & 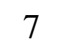 & 212 & 11 & 0.28 & 0.05 & 3. & 0 & 2 & 0 . & 1 & & 2 & 0.1 & 0.91 & 0. & 3 & 0 & 4 & & 266 & 20 \\
\hline $\mathrm{F}$ & 34 & 0 & 48 & 7 & 1 & 8 & $<\mathrm{LD}$ & & 11.50 & 0 & 1.6 & 0.7 & 11.6 & & 1.0 & & 0.8 & 0 & 1.5 & 0.2 & 177 & 12 & 338 & 70 \\
\hline ES2 & 2.34 & 5 & 29 & 3 & 105 & 5 & 0.03 & 0.05 & 5.53 & 0 & 1.0 & 0.5 & 5.2 & 0.2 & 1.02 & 0.05 & 1.1 & 0.3 & 0.67 & 0.09 & 33 & 0 & 170 & 14 \\
\hline ES3 & 2.49 & 0.06 & 48 & 3 & 203 & 9 & 0.6 & 0.2 & 7.37 & 0.05 & 1.0 & 0.6 & 8. & 0.3 & 1.27 & 0.06 & 1.2 & 0. & 2.4 & 0.3 & 79 & 4 & 363 & 31 \\
\hline ES4 & 2.00 & 0.05 & 142 & 18 & 136 & 5 & 0.2 & 0.1 & 9.54 & & 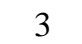 & 2 & 18.6 & 0.6 & 3.3 & & 1.6 & 0 . & 1.9 & 0.2 & 206 & 7 & 666 & 44 \\
\hline ES & 1.70 & & 38 & 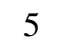 & 218 & 8 & 0.04 & 0.02 & 5.3 & & $J$ & 2 & 5 & 0.2 & 2.13 & 0.08 & 1.6 & 0 . & 2.3 & 0 & 12 & 4 & 134 & 55 \\
\hline F & 1 & 0.05 & 84 & 10 & 75 & 3 & $<\mathrm{LD}$ & & 14.33 & 0. & $<\mathrm{LD}$ & & 12 & 0 . & 0.88 & 0.05 & 1.2 & 0 . & 1.8 & 0 & 209 & 7 & 832 & 40 \\
\hline ES7 & 1.85 & & 72 & 10 & 266 & 10 & 0.31 & 0.05 & 5.71 & & ? & 2 & 9. & 0 & 4.2 & & 1.9 & 0. & 2.8 & 0. & 87 & 3 & 517 & 39 \\
\hline ES8 & 2.06 & 0.05 & 72 & 3 & 41 & 2 & 1.4 & 0.5 & 11.28 & & 0.9 & 0.6 & 11.2 & 0.4 & 1.50 & 0.05 & 0.8 & & 1.3 & 0.1 & 144 & 5 & 346 & 36 \\
\hline BA1 & 86 & 0 & 3.8 & 0.9 & 246 & 11 & 0.13 & 0.02 & 0.21 & 0.004 & 7 & 2 & 5.2 & 0.2 & 13.4 & 0.3 & 0.17 & 0.03 & 2.9 & 0.3 & 66 & 4 & 593 & 19 \\
\hline BA2 & & & 19 & 2 & 278 & 12 & 0.59 & 0.05 & 0 . & & 16.6 & 0.2 & 2.28 & 0.09 & 19.3 & 0.6 & 0.32 & 0.08 & 1.6 & 0 . & 115 & 0 & 938 & 25 \\
\hline MG1 & 2.39 & & & 14 & 225 & 17 & 1. & 0 & & & 10 & 2 & 5 & 1 & 132 & 4 & 2.0 & & 3.3 & & 263 & $1 /$ & 460 & 62 \\
\hline MG2 & 2.61 & & 157 & 6 & & 10 & 0.26 & 0.02 & & & 0.0 & 0. & & 2 & 2.01 & 0.09 & 1.68 & 0.06 & 1.8 & & 106 & & 554 & 17 \\
\hline RJ1 & 2.41 & 0.05 & 77 & 13 & 257 & 11 & 0.4 & 0.1 & 4.55 & 0.03 & 27.3 & 0.5 & 11.8 & 0.4 & 30.3 & 0.9 & 1.0 & 0.3 & 2.4 & 0.3 & 41 & 3 & 363 & 38 \\
\hline
\end{tabular}


Table 4: Pearson correlation coefficient obtained using the whole data set of elemental concentrations. Marked correlations are significant at

\begin{tabular}{|c|c|c|c|c|c|c|c|c|c|c|c|c|c|c|c|c|c|c|c|c|c|c|c|c|c|c|c|c|}
\hline & Th & $U$ & $\mathrm{~K}$ & As & $\mathrm{Ba}$ & $\mathrm{Br}$ & $\mathrm{Ca}$ & $\mathrm{Ce}$ & Co & $\mathrm{Cr}$ & Cs & $\mathrm{Eu}$ & $\mathrm{Fe}$ & $\mathrm{Hf}$ & $\mathrm{La}$ & $\mathrm{Lu}$ & $\mathrm{Na}$ & $\mathrm{Nd}$ & $\mathrm{Rb}$ & $\mathrm{Sb}$ & $\mathrm{Sc}$ & $\mathrm{Se}$ & $\mathrm{Sm}$ & $\mathrm{Ta}$ & $\mathrm{Tb}$ & $\mathrm{Yb}$ & $\mathrm{Zn}$ & $\mathrm{Zr}$ \\
\hline Th & 1.00 & & & & & & & & & & & & & & & & & & & & & & & & & & & \\
\hline$U$ & 0.31 & 1.00 & & & & & & & & & & & & & & & & & & & & & & & & & & \\
\hline $\mathrm{K}$ & 0.08 & 0.11 & 1.00 & & & & & & & & & & & & & & & & & & & & & & & & & \\
\hline As & -0.32 & -0.10 & -0.31 & 1.00 & & & & & & & & & & & & & & & & & & & & & & & & \\
\hline $\mathrm{Ba}$ & -0.01 & -0.34 & 0.49 & -0.21 & 1.00 & & & & & & & & & & & & & & & & & & & & & & & \\
\hline $\mathrm{Br}$ & 0.73 & -0.07 & 0.14 & 0.59 & 0.10 & 1.00 & & & & & & & & & & & & & & & & & & & & & & \\
\hline $\mathrm{Ca}$ & -0.04 & 0.02 & -0.57 & 0.16 & -0.24 & -0.16 & 1.00 & & & & & & & & & & & & & & & & & & & & & \\
\hline $\mathrm{Ce}$ & 0.69 & -0.21 & 0.45 & -0.35 & 0.60 & 0.63 & -0.24 & 1.00 & & & & & & & & & & & & & & & & & & & & \\
\hline Co & -0.09 & -0.30 & 0.05 & 0.35 & 0.03 & 0.38 & -0.07 & 0.00 & 1.00 & & & & & & & & & & & & & & & & & & & \\
\hline $\mathrm{Cr}$ & -0.03 & -0.04 & -0.35 & -0.17 & 0.00 & -0.46 & -0.07 & 0.03 & -0.37 & 1.00 & & & & & & & & & & & & & & & & & & \\
\hline Cs & 0.26 & 0.57 & 0.27 & 0.09 & -0.15 & -0.04 & -0.13 & -0.07 & -0.16 & -0.14 & 1.00 & & & & & & & & & & & & & & & & & \\
\hline Eu & 0.89 & -0.33 & 0.50 & -0.04 & 0.33 & 0.78 & -0.05 & 0.77 & -0.03 & -0.18 & -0.06 & 1.00 & & & & & & & & & & & & & & & & \\
\hline $\mathrm{Fe}$ & 0.08 & -0.27 & 0.08 & 0.67 & 0.49 & 0.38 & -0.16 & 0.29 & 0.08 & -0.01 & -0.02 & 0.32 & 1.00 & & & & & & & & & & & & & & & \\
\hline $\mathrm{Hf}$ & 0.37 & 0.07 & 0.25 & -0.18 & 0.23 & 0.70 & -0.47 & 0.41 & 0.15 & -0.08 & 0.07 & 0.10 & 0.09 & 1.00 & & & & & & & & & & & & & & \\
\hline La & 0.68 & -0.22 & 0.48 & -0.29 & 0.53 & 0.74 & -0.22 & 0.94 & 0.01 & -0.14 & -0.05 & 0.81 & 0.31 & 0.33 & 1.00 & & & & & & & & & & & & & \\
\hline Lu & 0.69 & 0.11 & -0.04 & -0.20 & 0.21 & -0.25 & -0.14 & 0.38 & -0.12 & 0.62 & 0.02 & -0.17 & -0.02 & 0.27 & -0.02 & 1.00 & & & & & & & & & & & & \\
\hline $\mathrm{Na}$ & 0.65 & 0.18 & 0.19 & 0.06 & -0.10 & 0.82 & -0.26 & 0.32 & -0.14 & -0.22 & 0.04 & 0.62 & 0.11 & 0.51 & 0.37 & -0.16 & 1.00 & & & & & & & & & & & \\
\hline $\mathrm{Nd}$ & 0.76 & -0.27 & 0.48 & -0.32 & 0.50 & 0.71 & -0.22 & 0.97 & 0.08 & -0.09 & -0.06 & 0.85 & 0.29 & 0.43 & 0.96 & 0.22 & 0.39 & 1.00 & & & & & & & & & & \\
\hline $\mathrm{Rb}$ & 0.81 & 0.28 & 0.18 & -0.34 & 0.12 & -0.19 & -0.35 & 0.37 & -0.13 & 0.50 & 0.25 & -0.21 & -0.24 & 0.38 & 0.05 & 0.85 & -0.06 & 0.24 & 1.00 & & & & & & & & & \\
\hline $\mathrm{Sb}$ & 0.78 & 0.16 & 0.06 & -0.14 & 0.12 & 0.57 & -0.01 & 0.55 & -0.21 & -0.12 & 0.40 & 0.81 & 0.29 & 0.01 & 0.59 & -0.17 & 0.54 & 0.61 & -0.11 & 1.00 & & & & & & & & \\
\hline Sc & 0.11 & -0.34 & 0.12 & 0.69 & 0.40 & 0.22 & -0.16 & 0.21 & 0.12 & -0.06 & -0.18 & 0.36 & 0.84 & -0.08 & 0.26 & -0.13 & 0.01 & 0.24 & -0.39 & 0.35 & 1.00 & & & & & & & \\
\hline $\mathrm{Se}$ & -0.13 & 0.16 & -0.12 & 0.07 & 0.31 & -0.07 & 0.23 & 0.08 & -0.12 & -0.05 & -0.14 & 0.41 & 0.08 & -0.08 & 0.18 & -0.15 & -0.01 & 0.00 & -0.15 & 0.45 & 0.08 & 1.00 & & & & & & \\
\hline $\mathrm{Sm}$ & 0.90 & -0.11 & 0.67 & -0.26 & 0.33 & 0.75 & -0.12 & 0.83 & -0.05 & -0.18 & 0.14 & 0.97 & 0.24 & 0.22 & 0.87 & -0.07 & 0.58 & 0.90 & 0.06 & 0.80 & 0.27 & 0.31 & 1.00 & & & & & \\
\hline $\mathrm{Ta}$ & -0.09 & 0.13 & -0.07 & 0.02 & 0.34 & -0.13 & 0.20 & 0.05 & -0.11 & 0.00 & -0.12 & 0.09 & 0.07 & -0.07 & 0.09 & -0.09 & -0.04 & -0.03 & -0.11 & 0.17 & 0.06 & 0.99 & 0.07 & 1.00 & & & & \\
\hline $\mathrm{Tb}$ & 0.96 & -0.06 & 0.46 & 0.13 & 0.12 & 0.72 & -0.09 & 0.73 & 0.01 & -0.09 & 0.07 & 0.92 & 0.17 & 0.26 & 0.71 & 0.37 & 0.59 & 0.81 & 0.41 & 0.75 & 0.24 & -0.04 & 0.93 & -0.06 & 1.00 & & & \\
\hline $\mathrm{Yb}$ & 0.32 & 0.33 & 0.15 & -0.31 & 0.03 & 0.01 & -0.26 & 0.31 & -0.15 & 0.37 & 0.12 & 0.12 & -0.21 & 0.24 & 0.12 & 0.65 & 0.09 & 0.23 & 0.65 & 0.24 & -0.17 & -0.05 & 0.24 & 0.01 & 0.38 & 1.00 & & \\
\hline $\mathrm{Zn}$ & 0.08 & -0.05 & 0.17 & 0.02 & -0.06 & -0.03 & -0.07 & -0.05 & -0.06 & -0.17 & -0.01 & 0.06 & -0.17 & -0.07 & -0.05 & -0.04 & 0.00 & -0.04 & -0.02 & 0.26 & 0.23 & -0.08 & 0.08 & -0.06 & 0.12 & 0.18 & 1.00 & \\
\hline $\mathrm{Zr}$ & 0.76 & 0.10 & 0.37 & -0.35 & 0.26 & 0.92 & -0.19 & 0.70 & 0.00 & -0.30 & 0.17 & 0.79 & 0.20 & 0.90 & 0.71 & 0.06 & 0.77 & 0.76 & 0.15 & 0.70 & 0.11 & 0.06 & 0.79 & -0.01 & 0.74 & 0.17 & -0.01 & 1.00 \\
\hline
\end{tabular}


Figure 4: Dendrogram for the variables measured in the analyzed ornamental rocks

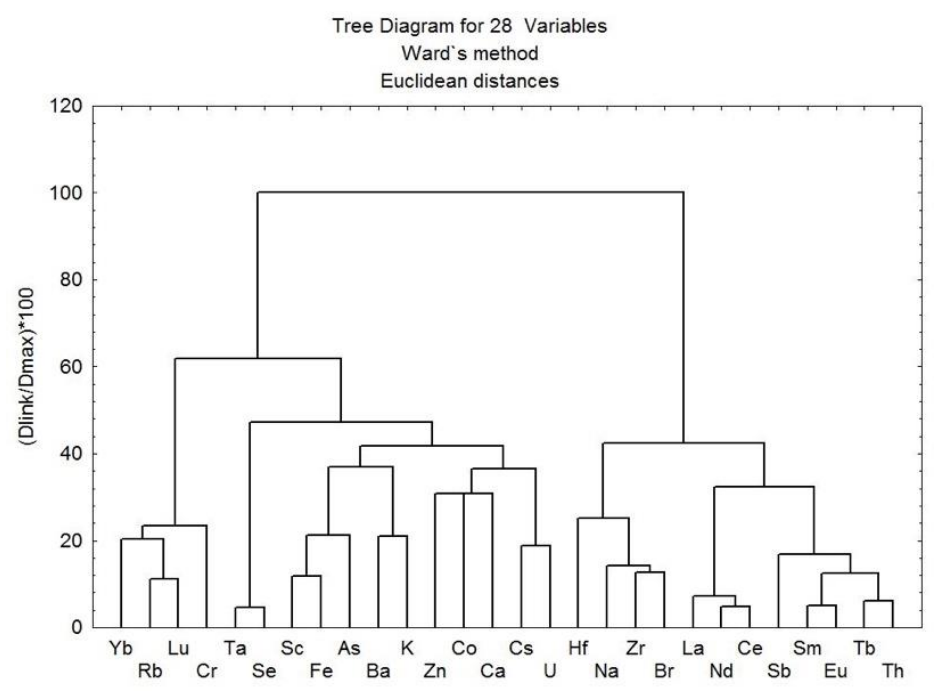

Figure 5: Factor analysis graph with the loading factors for factor 1 and 2, with the explained variance of each factor

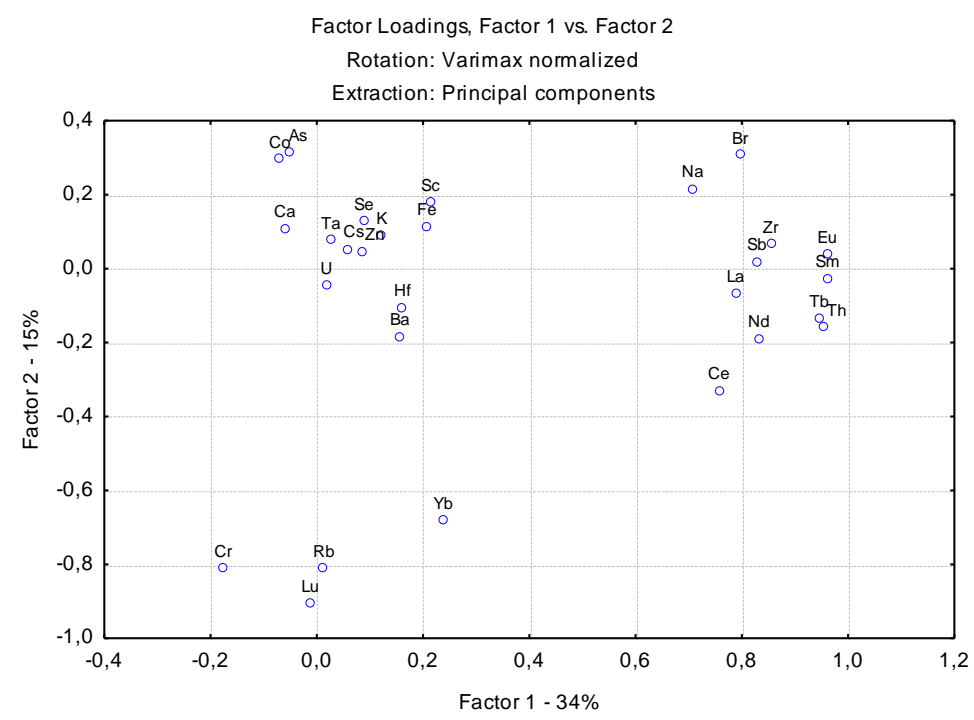

As sample $\mathrm{CH} 7$ showed the highest values for all radiological hazard indices determined, it will be discussed separately. Thus, citation to all samples means that $\mathrm{CH} 7$ is not included.

The range of Raeq varied from 5.5 to $775 \mathrm{~Bq} \mathrm{~kg}^{-1}$ in the samples CH9 and SP2, respectively, with a mean value of $299 \mathrm{~Bq} \mathrm{~kg}^{-1}$. Fig. 7 shows the mean values for samples from Switzerland and Brazil and also for Brazilian states. The mean value (and standard deviation) of Raeq in Swiss samples was $257 \pm 187 \mathrm{~Bq} \mathrm{~kg}^{-1}$ and for the Brazilian samples $325 \pm 175 \mathrm{~Bq} \mathrm{~kg}^{-1}$. Samples from Espírito 
Santo state (ES) were the ones with the lowest Raeq mean value. The recommended value of 370 $\mathrm{Bq} \mathrm{kg}^{-1}$ was exceeded in samples from São Paulo (SP), Rio de Janeiro (RJ) and Switzerland (CH). Sample CH7 exceeded this value 11.5 times. For a comparison purpose, Fig. 7 shows mean Raeq obtained in samples from Pakistan, PK, [33]; Egypt, EG, [34]; Jordan, JO, [35]; and with two other Brazilian assessments, BR2 [3] and BR3 [36]. As can be observed, Brazilian samples tend to present higher Raeq average values than those observed abroad. There was a good agreement in the mean values obtained in this study with the ones reported for other Brazilian samples.

Figure 6: Dendrogram obtained in the cluster analysis according to the rock classification

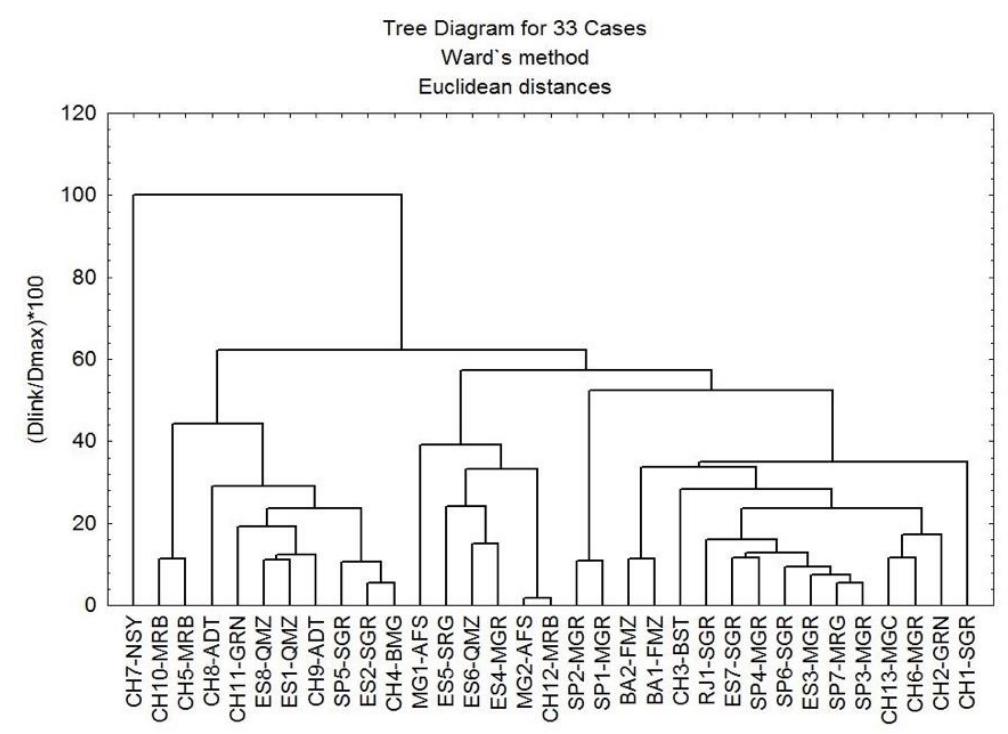

Figure 7: Radium equivalent, in Bq kg-1, according to the samples origin (this study: $C H, B R 1, S P$, $E S, B A, M G$ and $R J)$ and literature values $(P K, E G, B R 2, J O$ and $B R 3)$

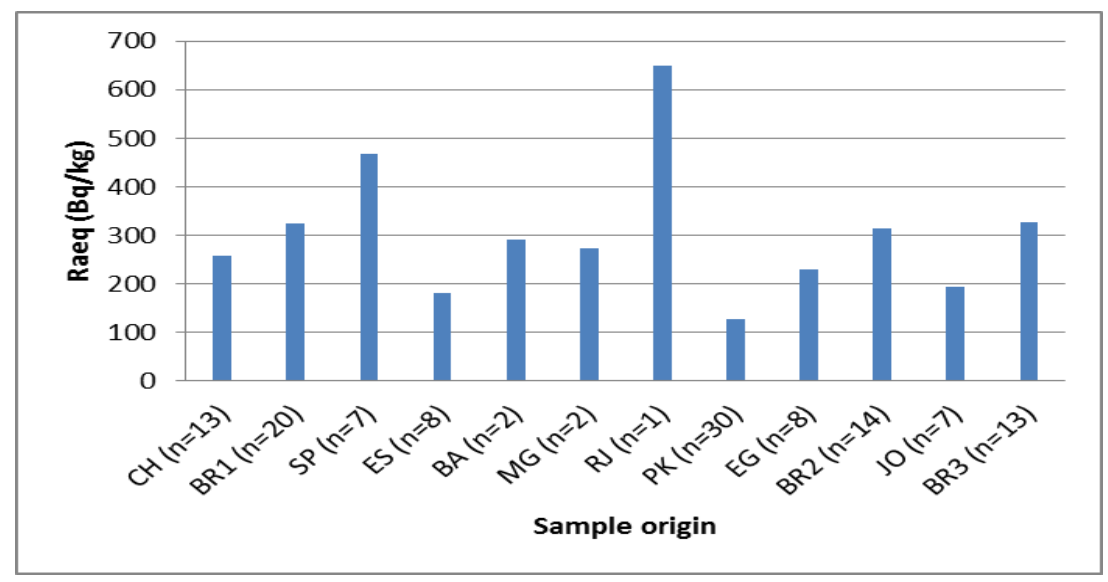


Table 5: Radiological hazard indices radium equivalent activity (Raeq), external hazard index (Hex), internal hazard index (Hin), absorbed dose rate (D), annual gonadal dose equivalent (AGDE), annual effective dose equivalent (AEDE) and excess life time cancer risk (ELCR).

\begin{tabular}{|c|c|c|c|c|c|c|c|c|c|c|c|c|c|c|}
\hline & \multicolumn{2}{|l|}{ Ra eq } & \multicolumn{2}{|l|}{$\mathrm{H}_{\mathrm{ex}}$} & \multicolumn{2}{|l|}{$\mathrm{H}_{\text {in }}$} & \multicolumn{2}{|l|}{$\mathrm{D}$} & \multicolumn{2}{|l|}{ AGDE } & \multicolumn{2}{|l|}{ AEDE } & \multicolumn{2}{|c|}{ ELCR } \\
\hline & $\mathrm{Bq} / \mathrm{kg}$ & & & & & & nGy/h & & $\mathrm{mSv} / \mathrm{y}$ & & $\mathrm{mSv} / \mathrm{y}$ & & $\mathrm{x} 10^{-3}$ & \\
\hline $\mathrm{CH} 1$ & 308 & \pm 49 & 0,41 & $\pm 0,02$ & 1.0 & \pm 0.1 & 148 & \pm 25 & 1.1 & \pm 0.2 & 0.7 & \pm 0.1 & 2.5 & \pm 0.4 \\
\hline $\mathrm{CH} 2$ & 359 & \pm 50 & 0,48 & $\pm 0,03$ & 1.2 & \pm 0.2 & 170 & \pm 26 & 1.2 & \pm 0.2 & 0.8 & \pm 0.1 & 2.9 & \pm 0.4 \\
\hline $\mathrm{CH} 3$ & 651 & \pm 36 & 0,88 & $\pm 0,05$ & 2.2 & \pm 0.1 & 298 & \pm 16 & 2.1 & \pm 0.1 & 1.46 & \pm 0.08 & 5.1 & \pm 0.3 \\
\hline $\mathrm{CH} 4$ & 112 & \pm 7 & 0,15 & $\pm 0,01$ & 0.35 & \pm 0.02 & 53 & \pm 3 & 0.38 & \pm 0.02 & 0.26 & \pm 0.02 & 0.91 & \pm 0.06 \\
\hline CH5 & 199 & \pm 11 & 0,27 & $\pm 0,02$ & 0.93 & \pm 0.05 & 90 & \pm 5 & 0.61 & \pm 0.03 & 0.44 & \pm 0.02 & 1.55 & \pm 0.08 \\
\hline CH6 & 403 & \pm 42 & 0,54 & $\pm 0,04$ & 1.6 & \pm 0.2 & 189 & \pm 20 & 1.3 & \pm 0.1 & 0.9 & \pm 0.1 & 3.2 & \pm 0.3 \\
\hline $\mathrm{CH} 7$ & 4241 & \pm 249 & 5,70 & $\pm 0,48$ & 11.5 & \pm 0.7 & 1791 & \pm 105 & 12.4 & \pm 0.7 & 8.8 & \pm 0.5 & 30.8 & \pm 1.8 \\
\hline $\mathrm{CH} 8$ & 148 & \pm 11 & 0,20 & $\pm 0,01$ & 0.63 & \pm 0.04 & 70 & \pm 5 & 0.49 & \pm 0.04 & 0.34 & \pm 0.02 & 1.20 & \pm 0.09 \\
\hline $\mathrm{CH} 9$ & 5.5 & \pm 0.4 & 0,0074 & $\pm 0,0007$ & 0.015 & \pm 0.001 & 2.3 & \pm 0.1 & 0.016 & \pm 0.001 & 0.011 & \pm 0.001 & 0.040 & \pm 0.003 \\
\hline $\mathrm{CH} 10$ & 37 & \pm 4 & 0,050 & $\pm 0,004$ & 0.19 & \pm 0.02 & 17 & \pm 2 & 0.12 & \pm 0.01 & 0.08 & \pm 0.01 & 0.29 & \pm 0.03 \\
\hline $\mathrm{CH} 11$ & 157 & \pm 18 & 0,21 & $\pm 0,02$ & 0.60 & \pm 0.06 & 71 & \pm 9 & 0.49 & \pm 0.06 & 0.35 & \pm 0.04 & 1.2 & \pm 0.2 \\
\hline $\mathrm{CH} 12$ & 257 & \pm 57 & 0,35 & $\pm 0,02$ & 0.7 & \pm 0.2 & 129 & \pm 30 & 0.9 & \pm 0.2 & 0.6 & \pm 0.1 & 2.2 & \pm 0.5 \\
\hline $\mathrm{CH} 13$ & 446 & \pm 44 & 0,60 & $\pm 0,03$ & 1.6 & \pm 0.1 & 206 & \pm 22 & 1.4 & \pm 0.2 & 1.0 & \pm 0.1 & 3.5 & \pm 0.4 \\
\hline SP1 & 600 & \pm 45 & 0,81 & $\pm 0,06$ & 1.7 & \pm 0.1 & 261 & \pm 21 & 1.8 & \pm 0.1 & 1.3 & \pm 0.1 & 4.5 & \pm 0.4 \\
\hline SP2 & 775 & \pm 65 & 1,04 & $\pm 0,07$ & 2.4 & \pm 0.2 & 344 & \pm 31 & 2.4 & \pm 0.2 & 1.7 & \pm 0.2 & 5.9 & \pm 0.5 \\
\hline SP3 & 427 & \pm 24 & 0,58 & $\pm 0,03$ & 1.41 & \pm 0.08 & 195 & \pm 11 & 1.37 & \pm 0.08 & 0.96 & \pm 0.05 & 3.3 & \pm 0.2 \\
\hline SP4 & 514 & \pm 35 & 0,69 & $\pm 0,05$ & 1.5 & \pm 0.1 & 228 & \pm 16 & 1.6 & \pm 0.1 & 1.12 & \pm 0.08 & 3.9 & \pm 0.3 \\
\hline SP5 & 276 & \pm 24 & 0,37 & $\pm 0,03$ & 0.87 & \pm 0.08 & 120 & \pm 11 & 0.83 & \pm 0.08 & 0.59 & \pm 0.06 & 2.1 & \pm 0.2 \\
\hline SP6 & 354 & \pm 20 & 0,48 & $\pm 0,03$ & 1.15 & \pm 0.07 & 165 & \pm 9 & 1.17 & \pm 0.07 & 0.81 & \pm 0.05 & 2.8 & \pm 0.2 \\
\hline SP7 & 336 & \pm 19 & 0,45 & $\pm 0,03$ & 1.05 & \pm 0.06 & 156 & \pm 9 & 1.10 & \pm 0.06 & 0.76 & \pm 0.04 & 2.7 & \pm 0.2 \\
\hline ES1 & 105 & \pm 18 & 0,141 & $\pm 0,007$ & 0.29 & \pm 0.06 & 54 & \pm 10 & 0.40 & \pm 0.07 & 0.26 & \pm 0.05 & 0.9 & \pm 0.2 \\
\hline ES2 & 140 & \pm 10 & 0,19 & $\pm 0,01$ & 0.40 & \pm 0.03 & 68 & \pm 5 & 0.49 & \pm 0.04 & 0.33 & \pm 0.02 & 1.16 & \pm 0.09 \\
\hline ES3 & 279 & \pm 16 & 0,38 & $\pm 0,02$ & 0.98 & \pm 0.06 & 133 & \pm 8 & 0.95 & \pm 0.06 & 0.65 & \pm 0.04 & 2.3 & \pm 0.1 \\
\hline ES4 & 209 & \pm 13 & 0,28 & $\pm 0,02$ & 0.61 & \pm 0.04 & 98 & \pm 6 & 0.70 & \pm 0.04 & 0.48 & \pm 0.03 & 1.7 & \pm 0.1 \\
\hline ES5 & 235 & \pm 14 & 0,32 & $\pm 0,01$ & 0.70 & \pm 0.04 & 113 & \pm 7 & 0.81 & \pm 0.05 & 0.55 & \pm 0.03 & 1.9 & \pm 0.1 \\
\hline ES6 & 114 & \pm 7 & 0,154 & $\pm 0,002$ & 0.31 & \pm 0.02 & 60 & \pm 3 & 0.45 & \pm 0.03 & 0.29 & \pm 0.02 & 1.03 & \pm 0.06 \\
\hline ES7 & 270 & \pm 15 & 0,36 & $\pm 0,02$ & 0.78 & \pm 0.04 & 127 & \pm 7 & 0.91 & \pm 0.05 & 0.62 & \pm 0.03 & 2.2 & \pm 0.1 \\
\hline ES8 & 89 & \pm 6 & 0,121 & $\pm 0,006$ & 0.30 & \pm 0.02 & 44 & \pm 3 & 0.31 & \pm 0.02 & 0.21 & \pm 0.02 & 0.75 & \pm 0.05 \\
\hline BA1 & 308 & \pm 50 & 0,42 & $\pm 0,02$ & 1.2 & \pm 0.2 & 152 & \pm 26 & 1.1 & \pm 0.2 & 0.7 & \pm 0.1 & 2.6 & \pm 0.5 \\
\hline BA2 & 274 & \pm 17 & 0,37 & $\pm 0,02$ & 1.01 & \pm 0.06 & 127 & \pm 8 & 0.89 & \pm 0.05 & 0.62 & \pm 0.04 & 2.2 & \pm 0.1 \\
\hline MG1 & 273 & \pm 43 & 0,37 & $\pm 0,03$ & 0.9 & \pm 0.2 & 135 & \pm 22 & 1.0 & \pm 0.2 & 0.7 & \pm 0.1 & 2.3 & \pm 0.4 \\
\hline MG2 & 273 & \pm 60 & 0,37 & $\pm 0,02$ & 0.8 & \pm 0.2 & 138 & \pm 32 & 1.0 & \pm 0.2 & 0.7 & \pm 0.2 & 2.4 & \pm 0.5 \\
\hline RJ1 & 649 & \pm 41 & 0,87 & $\pm 0,06$ & 2.3 & \pm 0.2 & 295 & \pm 19 & 2.1 & \pm 0.1 & 1.45 & \pm 0.09 & 5.1 & \pm 0.3 \\
\hline $\begin{array}{c}\mathrm{RV} \\
\text { or } \\
\mathrm{WM}^{\mathrm{a}}\end{array}$ & 370 & & $<1$ & & $<1$ & & 84 & & 0.36 & & 0.46 & & 1.16 & \\
\hline
\end{tabular}

${ }^{\mathrm{a}} \mathrm{RV}$ or $\mathrm{WV}$ : recommended value or worldwide average value. 
The external (Hex) and internal (Hin) hazard indices presented mean values of 0.38 and 0.995 , respectively considering all samples. The values ranged from 0.011 to 1.25 for Hex, in samples CH9 and SP2, respectively, and from 0.015 to 2.45 for Hin, in the same samples. Fig. 8 shows that the average values of both of these indices were slightly higher for the Brazilian samples than for the Swiss ones. Among the Brazilian states, the samples from São Paulo (SP), Espírito Santo (ES) and Bahia (BA) presented very close average values while Minas Gerais (MG) samples presented the lowest ones. Comparing with values in the literature, only the samples from MG present lower values than those observed in samples from abroad. Good agreement for this parameter was also found among the Brazilian samples analyzed in this study and the values (BR2) reported by Moura et al. [3]. Brazilian samples presented Hin average values higher than those from all other countries shown in Fig 8. Nevertheless high values of Hin were also found in building material by Bello et al. [37], whose measures varied from 0.23 to 3.99 for rocks, sediment and building samples in Southwestern Nigeria and by Alharbi et al. [38] who found values varying from 0.14 to 2.12 in granite rocks of Kingdom of Saudi Arabia.

Figure 8: External (Hex) and internal (Hin) hazard indices, according to the samples origin (this study: $C H, B R 1, S P, E S, B A, M G$ and RJ) and literature values (PK, EG, BR2 and JO)

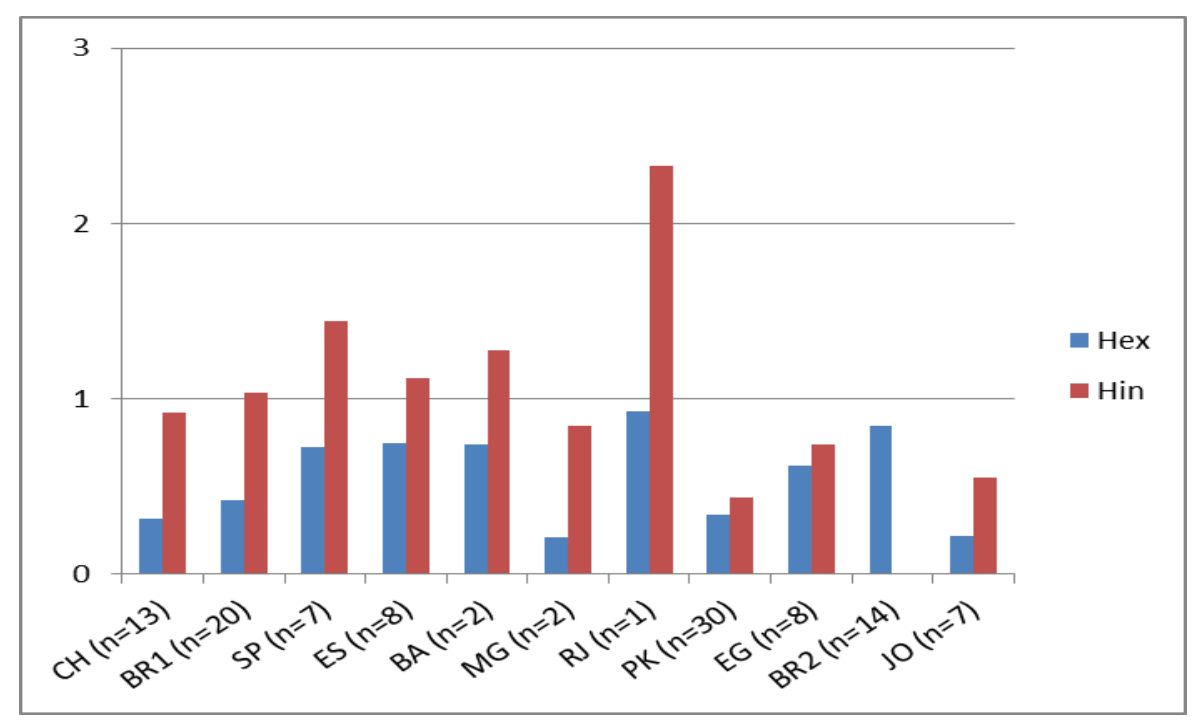

Considering the limit of 1 for Hex and Hin, the former was exceeded in two SP samples while the latter was exceeded in all SP samples but SP5, in BA and RJ samples, among the Brazilian ones. Among the Swiss samples Hex was not exceeded while Hin was higher than 1 in 5 samples. CH7 
presented values for external and internal hazard index 5.7 and 11.5 times higher than the recommended value, respectively.

The absorbed dose rate (D) ranged from 2.3 to $344 \mathrm{nGy} \mathrm{h}^{-1}$, with a mean value of $139 \mathrm{nGy} \mathrm{h}^{-1}$ considering all samples. The lowest value was measured in sample $\mathrm{CH} 9$ and the highest one in sample SP2. Fig 9 shows the comparison among the D values obtained in the samples analyzed in this study and also values reported in the literature. Among the Brazilian samples, the ones from São Paulo (SP) and Rio de Janeiro (RJ) showed the highest values, and the lowest ones were found in Espírito Santo (ES) samples. Brazilian average was higher than the one observed in Swiss samples and also higher than that reported for PK, JO and EG. The absorbed dose rate measured is quite similar to that found in granites analyzed by Anjos et al. [39]. Compared with the world average value of $58 \mathrm{nGy} \mathrm{h}^{-1}$ [11], only three Swiss and three Brazilian samples were below it, indicating that the majority of the analyzed samples are up to six times greater than the world average value. In the case of sample $\mathrm{CH} 7$ with a $\mathrm{D}$ of $1791 \mathrm{nGy} \mathrm{h}^{-1}$, the worldwide average value is exceeded by 31 times.

Figure 9: Absorbed dose rate, in $n G y$ h-1, according to the samples origin (this study: $C H, B R 1$, $S P, E S, B A, M G$ and $R J)$ and literature values (PK, EG, JO and BR4)

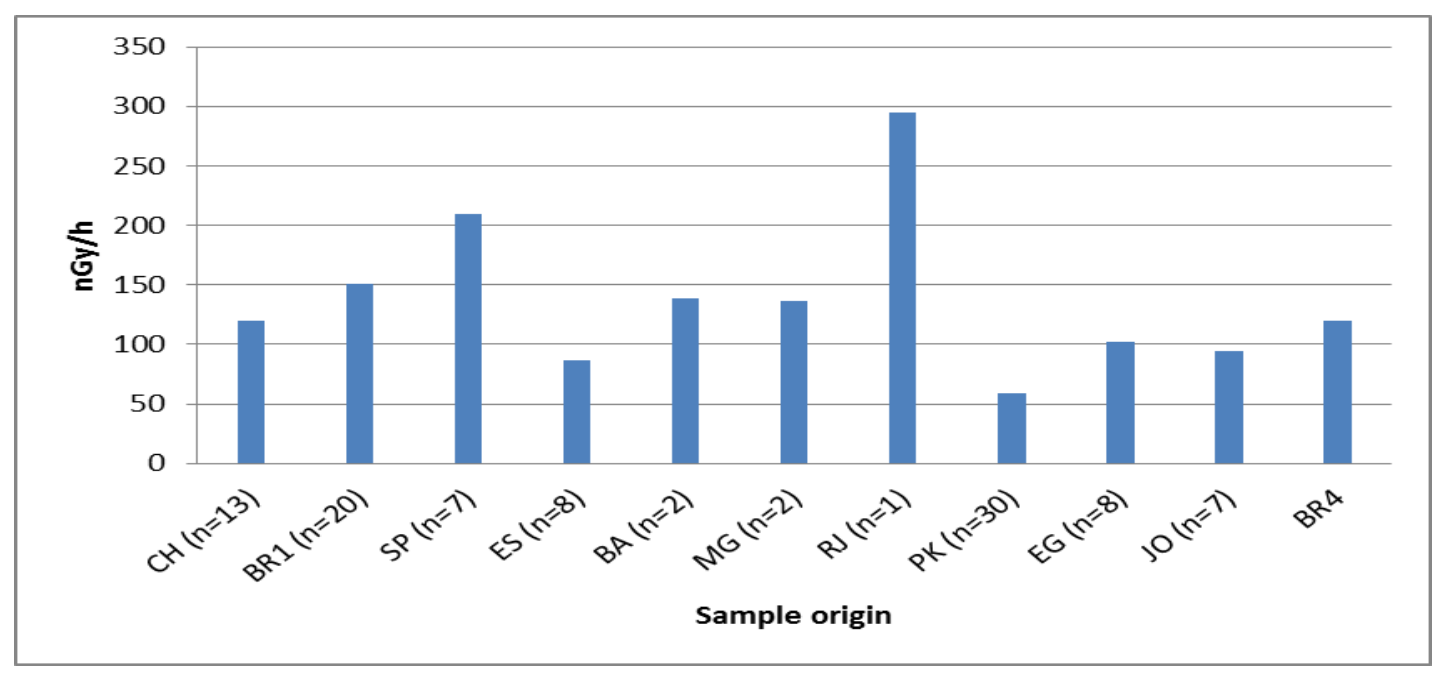

The annual gonadal dose equivalent (AGDE), annual effective dose equivalent (AEDE) and excess life time cancer risk (ELCR) indices presented mean values of $0.983 \mathrm{mSv} \mathrm{y}^{-1}, 0.683 \mathrm{mSv} \mathrm{y}^{-1}$ and 
2.389, respectively, for all samples. AGDE varied from 0.016 to $2.393 \mathrm{mSv} \mathrm{y}^{-1}$; AEDE varied from 0.011 to $1.685 \mathrm{mSv}^{-1}$ and ELCR varied from 0.040 to 5.899, all in samples CH9 and SP2, respectively. Fig 10 shows mean values for these parameters according to their origin and compared with the same values for literature in construction materials from Iraq (IQ) [40] and from Egypt (EG). The mean value of AGDE is higher for the Brazilian samples than that collected in Switzerland and those reported for Iraq and Egypt. Among the Brazilian samples the highest values were found in São Paulo (SP) and Rio de Janeiro (RJ) samples. Considering the average world value of $0.36 \mathrm{mSv} \mathrm{y}^{-1}$ for AGDE [12] (UNSCEAR, 1998) only two samples from Switzerland and three samples from Brazil have values below it. The values found in this study are up to 6.6 times higher than the world average, and sample $\mathrm{CH} 7$ exceeded this value by 34 times. Of the total, fifteen samples are greater than the established limit of $1 \mathrm{mSv} \mathrm{y}^{-1}$ [41].

Figure 10: Annual gonadal dose equivalent $(A G D E)$, annual effective dose equivalent (AEDE) and excess life time cancer risk (ELCR) according to the samples origin (this study: CH, BR1, SP, ES,

$B A, M G$ and $R J)$ and literature values (IQ and $E G$ )

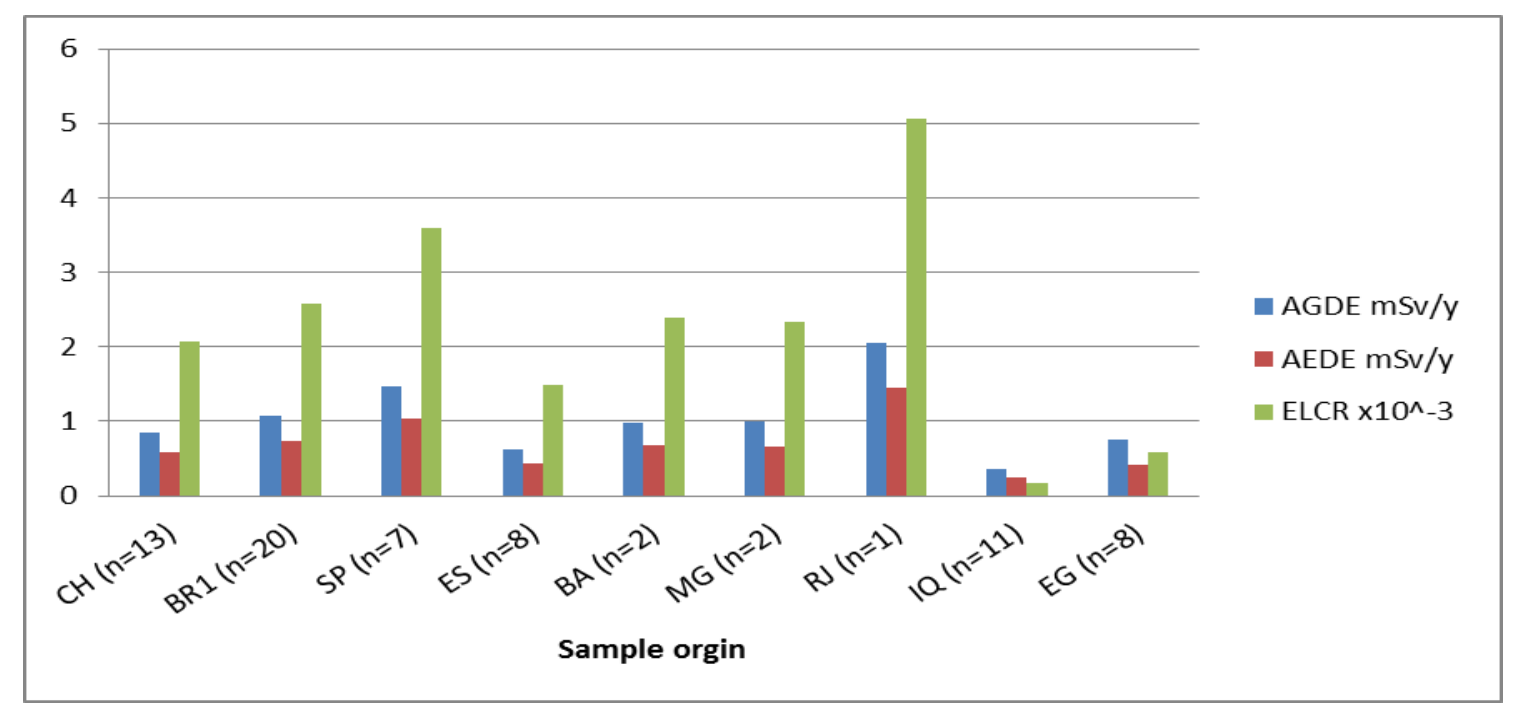

The results of AEDE were lower than the average annual indoor effective dose from terrestrial radionuclides of $0.41 \mathrm{mSv} \mathrm{y}^{-1}$ [42] in six samples from Switzerland and in five samples from Brazil. The measured values were up to 3.6 times higher than the worldwide average value and sample CH7 was 19 times greater than this average value. Six samples exceeded the limit of $1 \mathrm{mSv} \mathrm{y}^{-1}$. 
The results of ELCR were lower than the average global value in only three Swiss samples and in three samples from Brazil. The values above the average were up to five times higher and for sample $\mathrm{CH} 7,26$ times higher.

\subsection{Multivariate statistics of the radiological risk indices}

Cluster analysis was applied to evaluate the radiological indices among the analyzed samples considering the activity concentrations of ${ }^{232} \mathrm{Th},{ }^{238} \mathrm{U}$ and ${ }^{40} \mathrm{~K}$, and the radiological indices determined in this study. The result is showed in Fig. 11. From the CA result it is obvious that sample $\mathrm{CH} 7$ is highlighted, being the one with the highest values for all radiological risk indices determined among the analyzed samples.

Figure 11: Dendrogram obtained in cluster analysis considering the activity concentrations of the radionuclides and radiological risk indices

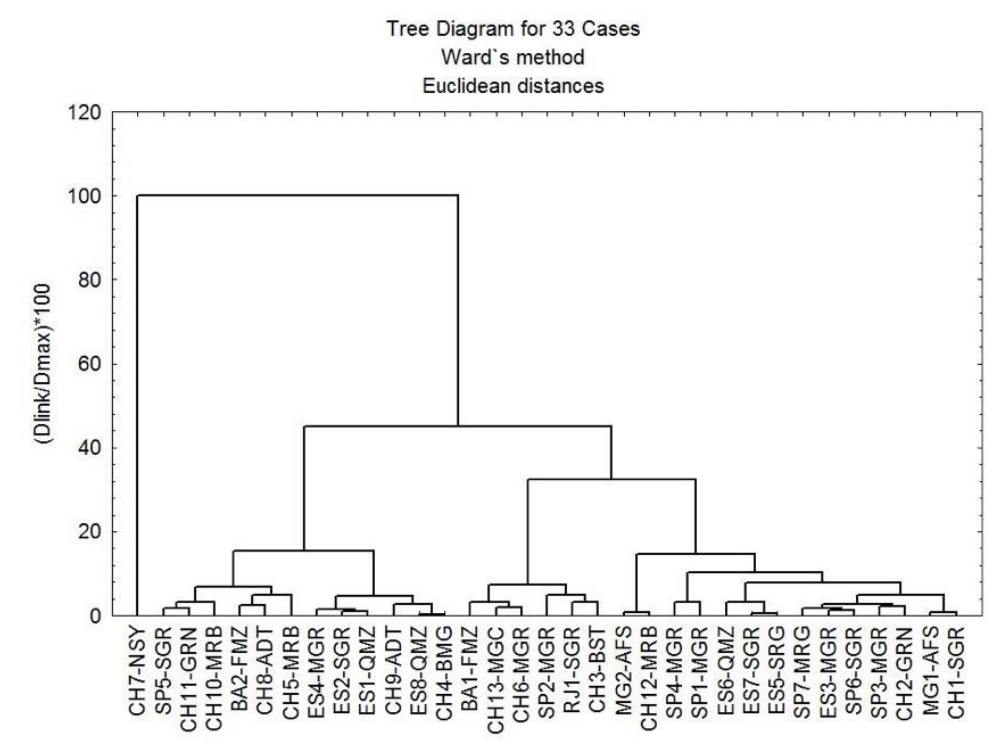

Cutting the dendrogram at a level of $20 \%$ of the $100 *$ Dlink/Dmax, three main groups (excluding sample CH7) were observed (Fig. 11). The basic statistics for each group is summarized in Table 6. 
Table 6: Basic statistics of the obtained groups using cluster analysis, considering the activity concentrations of the radionuclides and radiological indices.

\begin{tabular}{|c|c|c|c|c|c|c|c|c|}
\hline & Valid N & Mean & Median & Minimum & Maximum & Std.Dev. & Skewness & Kurtosis \\
\hline & \multicolumn{8}{|c|}{ Group 1} \\
\hline $\mathrm{Th}$ & 12 & 44.7 & 35.9 & 2.5 & 147.4 & 41.7 & 1.46 & 2.36 \\
\hline $\mathrm{U}$ & 11 & 49.7 & 31.6 & 3.9 & 143.8 & 44.4 & 1.07 & 0.37 \\
\hline K & 11 & 516.3 & 549.5 & 24.9 & 1041.0 & 365.4 & 0.01 & -1.43 \\
\hline $\mathrm{Ra}$ eq & 12 & 145.9 & 144.1 & 5.5 & 275.5 & 84.0 & 0.06 & -0.51 \\
\hline Hex & 12 & 0.19 & 0.16 & 0.011 & 0.47 & 0.14 & 0.69 & -0.19 \\
\hline Hin & 12 & 0.52 & 0.50 & 0.015 & 1.01 & 0.31 & 0.17 & -0.93 \\
\hline $\mathrm{D}$ & 12 & 67.8 & 68.8 & 2.3 & 126.6 & 37.5 & -0.09 & -0.36 \\
\hline AGDE & 12 & 0.48 & 0.49 & 0.016 & 0.89 & 0.26 & -0.14 & -0.27 \\
\hline AEDE & 12 & 0.33 & 0.34 & 0.011 & 0.62 & 0.18 & -0.09 & -0.36 \\
\hline \multirow[t]{2}{*}{ ELCR } & 12 & 1.16 & 1.18 & 0.040 & 2.17 & 0.64 & -0.09 & -0.36 \\
\hline & & \multicolumn{7}{|c|}{ Group 2} \\
\hline $\mathrm{Th}$ & 6 & 184.5 & 186.3 & 22.6 & 387.1 & 129.7 & 0.45 & -0.09 \\
\hline $\mathrm{U}$ & 6 & 164.4 & 157.5 & 130.9 & 213.7 & 30.2 & 0.80 & 0.05 \\
\hline K & 6 & 1435.8 & 1345.3 & 1182.2 & 1797.4 & 251.2 & 0.75 & -1.48 \\
\hline Ra eq & 6 & 538.8 & 547.5 & 308.4 & 775.5 & 179.4 & 0.02 & -1.74 \\
\hline Hex & 6 & 0.741 & 0.75 & 0.27 & 1.25 & 0.354 & 0.15 & -0.79 \\
\hline Hin & 6 & 1.90 & 1.91 & 1.23 & 2.45 & 0.497 & -0.22 & -2.12 \\
\hline $\mathrm{D}$ & 6 & 247.3 & 250.4 & 151.6 & 343.6 & 75.4 & -0.005 & -1.99 \\
\hline AGDE & 6 & 1.73 & 1.75 & 1.08 & 2.39 & 0.52 & 0.006 & -2.05 \\
\hline AEDE & 6 & 1.21 & 1.23 & 0.74 & 1.69 & 0.37 & -0.005 & -1.99 \\
\hline \multirow[t]{2}{*}{ ELCR } & 6 & 4.25 & 4.30 & 2.60 & 5.90 & 1.29 & -0.005 & -1.99 \\
\hline & & \multicolumn{7}{|c|}{ Group 3} \\
\hline $\mathrm{Th}$ & 14 & 117.3 & 88.5 & 11.2 & 355.7 & 95.4 & 1.63 & 2.33 \\
\hline $\mathrm{U}$ & 13 & 52.0 & 52.4 & 15.6 & 100.8 & 30.4 & 0.20 & -1.41 \\
\hline K & 14 & 1460.5 & 1349.0 & 598.5 & 2372.2 & 449.3 & 0.51 & 0.97 \\
\hline Ra eq & 14 & 328.5 & 293.3 & 114.2 & 600.2 & 121.5 & 0.79 & 1.20 \\
\hline Hex & 14 & 0.40 & 0.32 & 0.04 & 1.04 & 0.27 & 1.27 & 1.57 \\
\hline Hin & 14 & 1.02 & 0.99 & 0.31 & 1.74 & 0.37 & 0.18 & 0.34 \\
\hline $\mathrm{D}$ & 14 & 154.1 & 142.8 & 59.9 & 260.8 & 49.6 & 0.54 & 1.13 \\
\hline AGDE & 14 & 1.10 & 1.04 & 0.45 & 1.82 & 0.34 & 0.48 & 1.17 \\
\hline AEDE & 14 & 0.76 & 0.70 & 0.29 & 1.28 & 0.24 & 0.54 & 1.13 \\
\hline ELCR & 14 & 2.65 & 2.45 & 1.03 & 4.48 & 0.85 & 0.54 & 1.13 \\
\hline
\end{tabular}

Group 1 was formed by the samples with lower activity concentrations and radiological hazard indices, meaning they are the safest samples among the analyzed ones. The values of Skewness and 
Kurtosis, near zero and negative, respectively, indicate that these samples form an homogeneous group with normal distribution for the radiological indices. For these samples the radiological hazard indices are mainly related to ${ }^{232} \mathrm{Th}$ concentrations as can be seen in Fig. 12, with inverse correlation between ${ }^{238} \mathrm{U}$ and ${ }^{40} \mathrm{~K}$.

Group 2 was formed by the samples with the highest activity concentrations and radiological hazard indices. Thus, these are the less safe samples among the analyzed ones considering gamma exposure, indicating that care must be taken in their use. This group contains foid-monzodiorite, mylonitic gneiss with chlorite and muscovite, monzogranite, syenogranite and basaltite rock types from Switzerland, Bahia, São Paulo and Rio de Janeiro. The radiological hazard indices are mainly related to ${ }^{232} \mathrm{Th}$ activity concentration, as can be seen in Fig. 13 , with ${ }^{238} \mathrm{U}$ and ${ }^{40} \mathrm{~K}$ directly correlated. As group 1, this group is also an homogenous group with normal distribution for the radiological hazard indices.

Figure 12: Principal component analysis result with the loading factor for the first and second component for the group 1 of the dendrogram shown in Fig 11

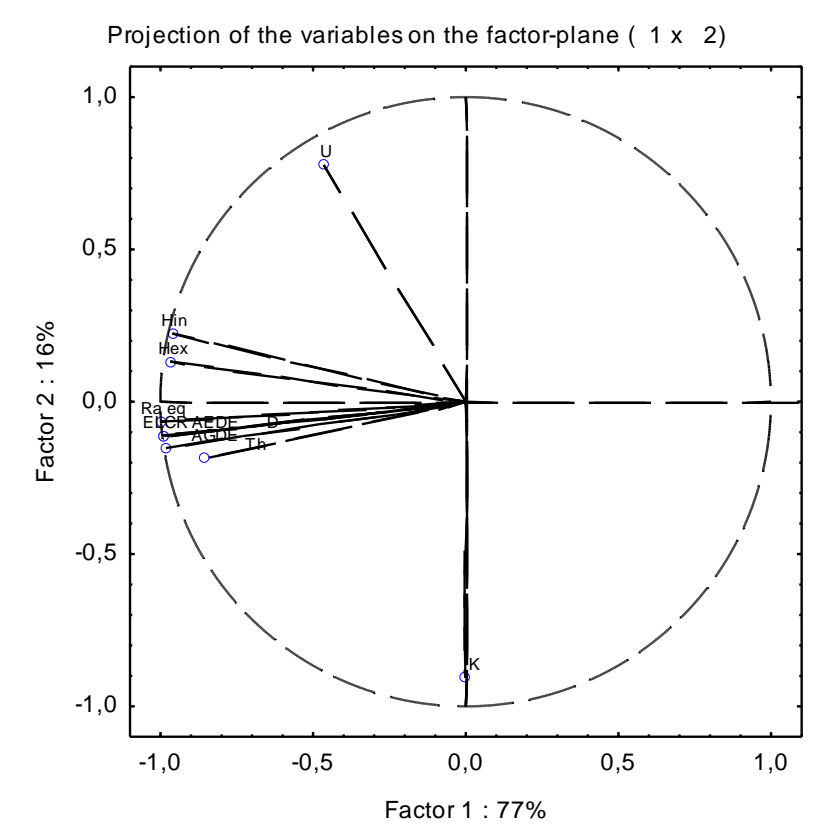

Group 3 presents the samples with intermediate values for the activity concentrations and radiological hazard indices. Nevertheless, only Raeq and Hex are within the limits for gamma external exposure, indicating that care must also be taken in their use. These samples form an 
heterogeneous group and the positive values of kurtosis indicate that the distribution is higher and narrower than the normal. Thorium content is also the main responsible for the observed radiological hazard indices, as shown in Fig. 14, also with negative correlation between ${ }^{238} \mathrm{U}$ and ${ }^{40} \mathrm{~K}$.

Figure 13: Principal component analysis result with the loading factor for the first and second component for the group 2 of the dendrogram shown in Fig. 11

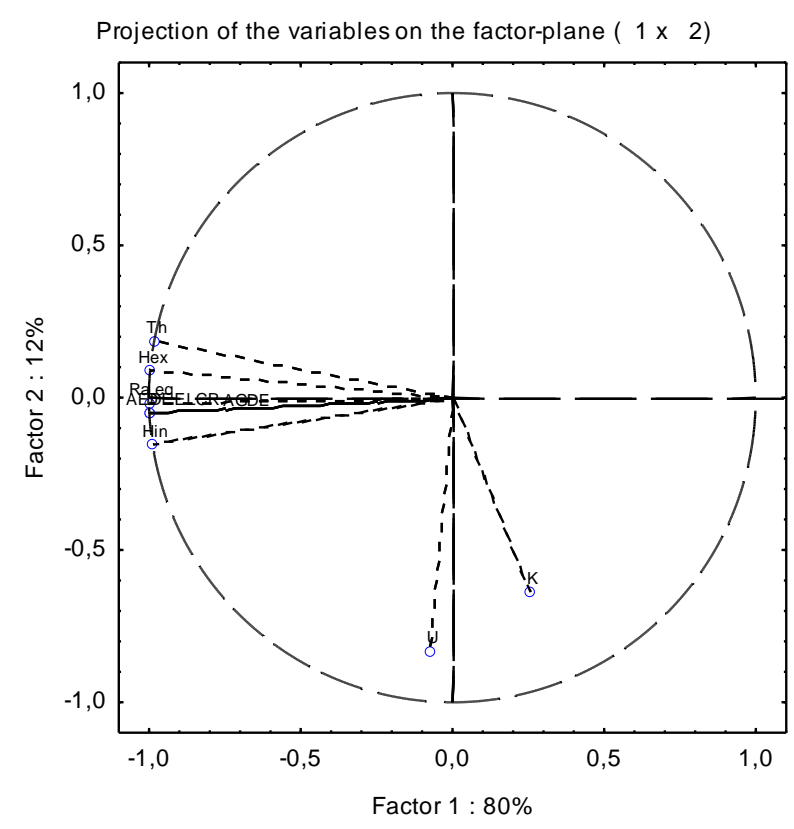

\section{CONCLUSION}

The analysis of ornamental rocks from Brazil and Switzerland showed that the activity concentrations of thorium, uranium and potassium covered a wide range of values, varying from 2.5 to $2966 \mathrm{~Bq} \mathrm{~kg}^{-1}$, from 3.9 to $214 \mathrm{~Bq} \mathrm{~kg}^{-1}$ and 25 to $2372 \mathrm{~Bq} \mathrm{~kg}^{-1}$, respectively. The highest ${ }^{232} \mathrm{Th}$ activity concentration was found for sample $\mathrm{CH} 7$, from Switzerland; the highest ${ }^{238} \mathrm{U}$ activity concentration was measured in RJ1 sample from Rio de Janeiro, Brazil; and for ${ }^{40} \mathrm{~K}$, in the sample MG2, from Minas Gerais, Brazil. The mean activity concentration and standard deviation for all 
Brazilian and Swiss samples were: $62 \pm 55 \mathrm{~Bq} \mathrm{~kg}^{-1}$ and $93 \pm 60 \mathrm{~Bq} \mathrm{~kg}^{-1}$ for ${ }^{238} \mathrm{U} ; 1126 \pm 516 \mathrm{~Bq} \mathrm{~kg}^{-}$ ${ }^{1}$ and $1005 \pm 778 \mathrm{~Bq} \mathrm{~kg}^{-1}$ for ${ }^{40} \mathrm{~K} ; 122 \pm 111 \mathrm{~Bq} \mathrm{~kg}^{-1}$ and $293 \pm 806 \mathrm{~Bq} \mathrm{~kg}^{-1}$ for ${ }^{232} \mathrm{Th}$, respectively.

Figure 14: Principal component analysis result with the loading factor for the first and second component for the group 3 of the dendrogram showed in Fig. 11

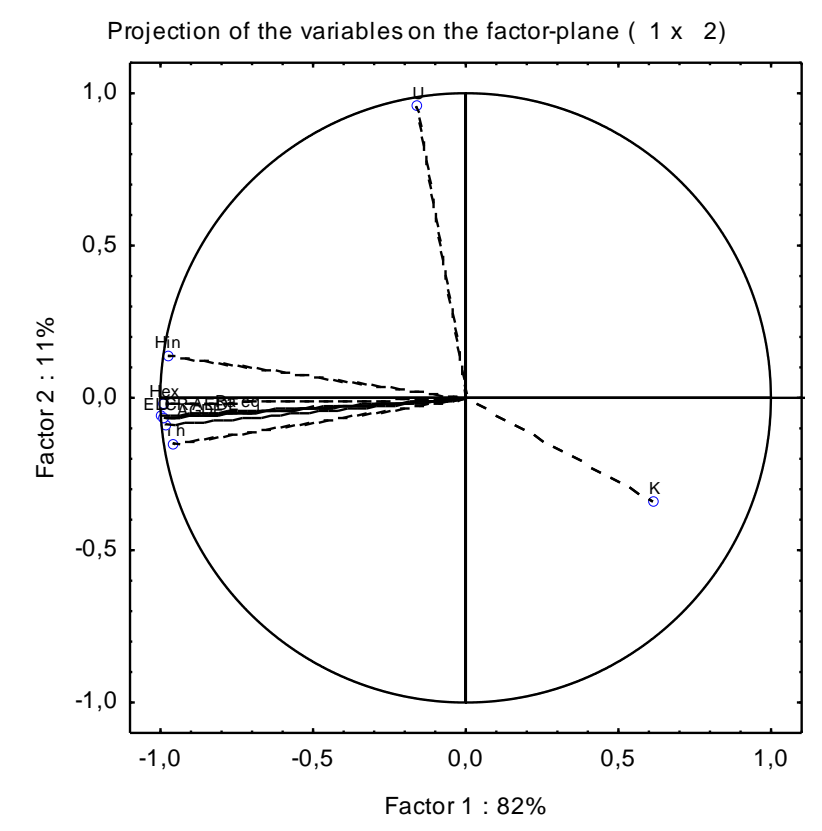

Brazilian ornamental rocks tend to be enriched in Th with a Th/U ratio varying from 5 to 15 . Swiss samples possess this ratio closer to the global average, 3 to 4 . Thorium was well correlated with rare earth elements (REE), Br, Hf, $\mathrm{Na}, \mathrm{Rb}, \mathrm{Sb}$ and $\mathrm{Zr}$; uranium only with $\mathrm{Cs}$; and $\mathrm{K}$ presented good correlations with $\mathrm{Ba}, \mathrm{REE}, \mathrm{Zr}$ and a negative correlation with $\mathrm{Ca}$. These findings indicate that $\mathrm{Th}$, $\mathrm{Zr}$, and REE may probably be associated with minerals such as zircon and apatite. Multivariate statistical analysis indicated that the variance observed in the analyzed rocks is more related to their petrographic classification than their provenance.

The radiological hazard index radium equivalent activity $\left(\mathrm{Ra}_{\mathrm{eq}}\right)$, external hazard index $\left(\mathrm{H}_{\mathrm{ex}}\right)$, internal hazard index $\left(\mathrm{H}_{\mathrm{in}}\right)$, absorbed dose rate (D), annual gonadal dose equivalent (AGDE), annual effective dose equivalent (AEDE) and excess life time cancer risk (ELCR) were exceeded in about two thirds of the Brazilian and Swiss samples. Results indicated that samples $\mathrm{CH} 4, \mathrm{CH} 5, \mathrm{CH} 8$, $\mathrm{CH} 9, \mathrm{CH} 10, \mathrm{CH} 11, \mathrm{SP} 5, \mathrm{ES} 1, \mathrm{ES} 2, \mathrm{ES} 4, \mathrm{ES} 8$ and BA2 are the safest ones considering gamma 
exposure. All the other samples should not be used in large amounts, mainly in the parts of the house where people can stand for long periods such as the rooms.

\section{ACKNOWLEDGMENT}

The authors wish to thank the National Council of Technological and Scientific Development (CNPq), São Paulo Research Foundation (FAPESP) from Brazil and Institute of Radiophysics (IRA) of Vaudois University Hospital Center (CHUV) from Switzerland for the technical cooperation.

\section{REFERENCES}

[1] ABIROCHAS. Perfil de Rochas Ornamentais e de Revestimento. Available in: http://www.ivolution.com.br/mais/fotos/6/18/3606/Exporta_Importa_12_2015.pdf. Access on: 22 Jan. 2016. Last accessed: 2015. (in Portuguese)

[2] NCS - Natural Stone Council. Granite Dimensional Stone Quarrying and Processing: A LifeCycle Inventory. University of Tennessee - Center of Clean Products. Available in: http://www.naturalstonecouncil.org/content/file/LCI\%20Reports/Granite_LCIv1.pdf. 2008. Last accessed: 11 Jul. 2016.

[3] MOURA, C. L., ARTUR, A. C., BONOTTO, D. M., GUEDES, S, MARTINELLI, C.D. Natural radioactivity and radon exhalation rate in Brazilian igneous rocks. Appl Radiat Isot. v. 69, p. 1094-1099, 2011.

[4] EC - EUROPEAN COMMISSION. Radiological Protection Principles concerning the Natural Radioactivity of Building Materials. Radiation protection No 112. Luxembourg. 1999.

[5] IAEA - TECDOC - Practical aspects of operating a neutron analysis laboratory. International Atomic Energy Agency, Vienna, 1999.

[6] NPL REPORT IR 6. Recommended Nuclear Decay Data. Available in: http://publications.npl.co.uk/npl_web/pdf/ir6.pdf. 2008. Last accessed: 04 October 2016. 
[7] TUFAIL, M. Radium equivalent activity in the light of UNSCEAR report. Environ Monit Assess, v. 184, p. 5663-5667, 2012.

[8] BERETKA, J., MATTHEW, P. J. Natural radioactivity of Australian building materials, industrial waste sand by-products. Health Phys. v. 48, p. 87-95, 1985.

[9] HEWAMANNA, R., SUMITHRARACHCHI, C., MAHAWATTE, P., NANAYAKKARA, H., RATNAYAKE, H. Natural radioactivity and gamma dose from Sri Lankan clay bricks used in building construction. Appl Radiat Isot. v. 54, p. 365-369, 2001.

[10] KRIEGER, R. Radioactivity of construction materials. Betonwerk Fertigteil Technol. v. 47, p. 468-473, 1981.

[11] UNSCEAR. Sources and Effects of Ionizing Radiation. Volume I: Effects. UNSCEAR 2008 Report. United Nations Scientific Committee on the Effects of Atomic Radiation, Report to the General Assembly, with scientific annexes. United Nations sales publication E.00.IX.4. United Nations, New York, 2010.

[12] RAMASAMY, V., PARAMASIVAM, K., SURESH, G., JOSE, M. T. Function of minerals in the natural radioactivity level of Vaigai River sediments, Tamilnadu, India. Spectroscopical approach. Spectrochim Acta A. v. 117(3), p. 340-350, 2014.

[13] UNSCEAR. Effects and risks of ionizing radiation. United Nations, New York, 1988. p 565571.

[14] CHANDRASEKARAN, A., RAVISANKAR, R., SENTHILKUMAR, G., THILlAIVELAVAN, K., DHINAKARAN, B., VIJAYAGOPAL, P., BRAMHA, S. N., VENKATRAMAN, B. Spatial distribution and lifetime cancer risk due to gamma radioactivity in Yelagiri Hills, Tamilnadu, India, Egypt J Basic and Appl Sci. v.1(1), p. 38-48, 2014.

[15] RAVISANKAR, R., VANASUNDARI, K., SUGANYA, M., RAGHU, Y., RAJALAKSHMI, A., CHANDRASEKARAN, A., SIVAKUMAR, S., CHANDRAMOHAN, J., VIJAYAGOPAL, P., VENKATRAMAN, B. Multivariate statistical analysis of radiological data of building materials used in Tiruvannamalai, Tamilnadu, India, Appl Radiat Isot. v. 85, p. 114-127, 2014.

[16] SINGH, J, SINGH, H, SINGH, S, BAJWA, BS, SONKAWADE, RG Comparative study of natural radioactivity levels in soil samples from the Upper Siwaliks and Punjab, India using gamma-ray spectrometry. J Environ Radioact. v. 100, p. 94-98, 2009. 
[17] TASKIN, H., KARAVUS, M., AY, P., TOPUZOGLU, A., HINDIROGLU, S., KARAHAN, G. Radionuclide concentrations in soil and life time cancer risk due to the gamma radioactivity in Kirklareli, Turkey. J Environ Radioact. v. 100, p. 49-53, 2009.

[18] ICRP. Radiation dose to patients from radiopharmaceuticals. Addendum 3 to ICRP Publication 53. ICRP Publication 106. Ann. ICRP 38, 2008. p 1-2.

[19] DEVORE, J.L. (1995) Probability and Statistics for Engineering and the Sciences, fourth ed. Duxbury Press, Belmont, CA.

[20] HUPP, A. M., MARShAlL, L. J., CAMPBELL, D. I., SMITH, R. W., MCGUFFIN, V. L. Chemometric analysis of diesel fuel for forensic and environmental applications. Anal Chim Acta, v. 606(2), p. 159-171, 2008.

[21] OTTO, M. Multivariate methods. In Kellner, R, Mermet, JM, Otto, M, Widmer, HM (Eds.), Analytical Chemistry. Weinheim: Wiley VCH. 1998.

[22] EINAX, J. W., ZWANZIGER, H. W., GEIB, S. Chemometrics in environmental analysis. Weinheim: Wiley. 1997.

[23] GEMPERLINE, P. J. Practical Guide to Chemometrics. Second ed. CRC Press, Boca Raton, FL. 2006.

[24] BUCCiAnti, A., APOllaro, C., Bloise, A., DE ROSA, R., FAlCONE, G., SCARCIGLIA, F., TALLARICO, A., VECCHIO, G. Natural radioactivity levels (K, Th, U and Rn) in the Cecita Lake area (Sila Massif, Calabria, Southern Italy): An attempt to discover correlations with soil features on a statistical base, Geoderma, v. 152 (1-2), p. 145-156, 2009.

[25] PLANT, J.A., REEDER, S., SALMINEN, R., SMITH, D. B., TARVAINEN, T., DE VIVO, B., PETTERSON, M. G. The distribution of uranium over Europe: geological and environmental significance. Transactions of the Institution of Mining and Metallurgy section B Appl Earth Sci. v. 112 (3), p. 221-238, 2003.

[26] KYSER K., CUNEY M. Geochemical characteristics of uranium and analytical methodologies. pp. 23-55 in. In: Cuney, M., Kyser, K., editors. Recent and Not-So-Recent Developments in Uranium Deposits and Implications for Exploration, Short Course Series 39. Mineralogical Association of Canada. 2008.

[27] LAUBENSTEIN, M., MAGALDI, D. Natural radioactivity of some red Mediterranean soils, Catena, v. 76(1), p. 22-26, 2008. 
[28] UNSCEAR. United Nations Scientific Committee on the Effects of Atomic Radiation, 1993. Sources and Effects of Ionizing Radiation, vol. I. United Nations, New York. 1993.

[29] NAGASAWA, H. Rare earth concentrations in zircons and apatites and their host dacites and granites, Earth and Planet Sc Lett, v. 9(4), p. 359-364, 1970.

[30] EL-ARABI, A. M. 226Ra, 232Th and 40K concentrations in igneous rocks from eastern desert, Egypt and its radiological implications, Radiat Meas, v. 42(1), p. 94-100, 2007.

[31] SMITH, M. P., MOORE, K., KAVECSÁNSZKI, D., FINCH, A. A., KYNICKY, J., WALL, F. From mantle to critical zone: A review of large and giant sized deposits of the rare earth elements, Geosci Front, v. 7(3), p. 315-334, 2016.

[32] ADAMS, J. A. S., OSMOND, J. K., ROGERS, J. J. W. The geochemistry of thorium and uranium, Phys Chem Earth, v. 3, p. 298-348, 1959.

[33] RAFIQUE, M., KHAN, A. R., JABBAR, A., RAHMAN, S. U., KAZMI, S. J. A., NASIR, T, ARSHED, W., MATIULLAH. Evaluation of radiation dose due to naturally occurring radionuclides in rock samples of different origins collected from Azad Kashmir, Russ Geol Geophys. v. 55(9), p. 1103-1112, 2014.

[34] DARWISH, D. A. E, ABUL-NASR, K. T. M., EL-KHAYATT, A. M. The assessment of natural radioactivity and its associated radiological hazards and dose parameters in granite samples from South Sinai, Egypt, J Radiat Res Appl Sci. v. 8(1), p. 17-25, 2015.

[35] SHARAF, J. M., HAMIDEEN, M. S. Measurement of natural radioactivity in Jordanian building materials and their contribution to the public indoor gamma dose rate, Appl Radiat Isot, v. 8, p. 61-66, 2013.

[36] SANTOS JÚNiOR, J. A., AMARAL, R. S., SILVA, C. M., MENEZES, R. S. C. Radium equivalent and annual effective dose from geological samples from Pedra - Pernambuco - Brazil, Radiat Meas, v. 45(7), p. 861-864, 2010.

[37] BELLO, I. A., JIBIRI, N. N., MOMOH, H. A. Determination of External and Internal Hazard Indices from Naturally Occurring Radionuclide in Rock, Sediment and Building Samples collected from Sikiti, Southwestern Nigeria. J Nat Sci Res, v. 4(12), p. 74 - 81, 2014.

[38] ALHARBI, W. R., ALZAHRANI, J. H., ABBADY, A. G. E. Assessment of Radiation Hazard Indices from Granite Rocks of the Southeastern Arabian Shield, Kingdom of Saudi Arabia, Aust J Basic Appl Sci, v. 5(6), p. 672-682, 2011. 
[39] ANJOS, R. M., JURI AYUB, J., CID, A. S., CARDOSO, R., LACERDA, T. External gammaray dose rate and radon concentration in indoor environments covered with Brazilian granites, $\mathbf{J}$ Environ Radioact, v. 102(11), p. 1055-1061, 2011.

[40] NAJAM, L. A., TAWFIQ, N. F, YOUNIS, A. S. Measurement of natural radioactivity in brick samples used in the construction in Iraq, Arch Phys Res, v. 6 (1), p. 13-19, 2015.

[41] ICRP. Recommendations of the International Commission on Radiological Protection. ICRP Publication 60. Oxford: Pergamon Press. 1991.

[42] ISINKAYE, M. O., EMELUE, H. U. Natural radioactivity measurements and evaluation of radiological hazards in sediment of Oguta Lake, South East Nigeria, J Radiat Res Appl Sci, v. 8(3), p. 459-469, 2015. 